\title{
Glycogen-Nucleic Acid Constructs for Gene Silencing in Multicellular Tumor Spheroids
}

Marcin Wojnilowicz ${ }^{1}$, Quinn A. Besford ${ }^{1}$, Yun-Long $\mathrm{Wu}^{2}$, Xian Jun Loh $^{3,4}$, Julia A. Braunger $^{1}$, Agata Glab ${ }^{1}$, Christina Cortez-Jugo ${ }^{1}$, Frank Caruso ${ }^{* 1}$ and Francesca Cavalieri*1,5

${ }^{1}$ ARC Centre of Excellence in Convergent Bio-Nano Science and Technology, and the Department of Chemical Engineering, The University of Melbourne, Parkville, Victoria 3010, Australia

${ }^{2}$ Fujian Provincial Key Laboratory of Innovative Drug Target Research and State Key Laboratory of Cellular Stress Biology, School of Pharmaceutical Sciences, Xiamen University, Xiamen, P. R. China

${ }^{3}$ Institute of Materials Research and Engineering, A*STAR (Agency for Science, Technology and Research), 2 Fusionopolis Way, Innovis, \#08-03, Singapore 138634, Singapore

${ }^{4}$ Department of Materials Science and Engineering, National University of Singapore, 9 Engineering Drive 1, Singapore 117576, Singapore

${ }^{5}$ Dipartimento di Scienze e Tecnologie Chimiche, Universita' degli Studi di Roma "Tor Vergata", via della ricerca scientifica 1, 00133 Rome, Italy

*Corresponding authors:

E-Mail: francesca.cavalieri@unimelb.edu.au.

E-Mail: fcaruso@unimelb.edu.au. 


\begin{abstract}
The poor penetration of nanocarrier-siRNA constructs into tumor tissue is a major hurdle for the in vivo efficacy of siRNA therapeutics, where the ability of the constructs to permeate the 3D multicellular matrix is determined by their physicochemical properties. Herein, we optimized the use of soft glycogen nanoparticles for the engineering of glycogen-siRNA constructs that can efficiently penetrate multicellular tumor spheroids and exert a significant gene silencing effect. Glycogen nanoparticles from different bio-sources and with different structural features were investigated. We show that larger glycogen nanoparticles ranging from 50 to $80 \mathrm{~nm}$ are not the optimal systems for complexation of nucleic acids if fine control of the size of constructs is required. Our studies suggest that $20 \mathrm{~nm}$ glycogen nanoparticles are optimal for complexation and efficient delivery of siRNA. The chemical composition, surface charge, and size of glycogen-siRNA constructs were finely controlled to minimize interactions with serum proteins and allow penetration into 3D multicellular spheroids of human kidney epithelial cells and human prostate cancer cells. We introduced $\mathrm{pH}$ sensitive moieties within the construct to enhance the early endosome escape and efficiently improve the silencing effect in vitro. Glycogen-siRNA constructs were found to mediate gene silencing in 3D multicellular spheroids causing $\sim 60 \%$ specific gene silencing. The optimized construct exhibited an in vivo circulation lifetime of $8 \mathrm{~h}$ in mice, with preferential accumulation in the liver. No accumulation in the kidney, lung, spleen, heart or brain or signs of toxicity in mice were observed. Our results highlight the potential for screening siRNA nanocarriers in 3D cultured prostate tumor models, thereby improving the predictive therapeutic efficacy of glycogen-based platforms in human physiological conditions.
\end{abstract}

Keywords: glycogen nanoparticles, RNA interference, multicellular tumor spheroids, in vivo biodistribution, prostate cancer 


\section{Introduction}

RNA-based therapeutics, such as small interfering RNA (siRNA), micro RNA (miRNA), and CRISPR-Cas9, have emerged as promising methods to alter gene function [1,2] in the treatment of diseases, including cancer [3-5] and neurodegenerative disorders [6]. Naked RNAs are rapidly cleared from the blood in vivo by the kidneys and liver, or degraded by serum nucleases. Furthermore, because of their negative charge, naked RNAs are too large and hydrophilic to cross cell membranes, and when in circulation they stimulate the innate immune system that triggers inflammatory responses [7]. Consequently, systemic administration of siRNA-based therapeutics requires an effective delivery agent such as a nanocarrier. Early delivery approaches evaluated in clinical trials include the encapsulation of siRNA in lipid-based nanoparticles, packaging in polymer-based nanocarriers, and chemical conjugation of siRNA with targeting $\mathrm{N}$-acetylgalactosamine tris GalNAc domains $[1,2,6]$. However, these formulations have shown toxicity and activated innate immune responses, and have limited metabolic stability and activity [8]. Hence, to date, no siRNA therapeutic for systemic administration has been commercialized, to our best knowledge $[1,2,8]$. The discrepancies between siRNA therapeutic efficacies obtained in animal models and outcomes of clinical trials in human diseases can be attributed to a lack of clinical relevance of the animal cancer models used for drug testing [9].

Lessons learnt for the future generation of RNA-based therapeutics is that the delivery systems and mechanism should be prioritized at the early stage of drugs development $[10,11,12]$. The optimal design of the nanocarriers in terms of physicochemical properties, specific and non-specific interactions in the complex biological microenvironment, biodistribution, the extracellular and intracellular trafficking of the cargo [13], are critical factors for the successful translation of RNA based therapies [8].

The predominant biological barriers faced by siRNA-nanocarriers are their stability during circulation against nuclease and serum proteins, secretion by the kidneys, immune system recognition and activation, extravasation and penetration into tissues. Furthermore, on the cellular level, barriers include adequate cellular uptake, endosomal escape, and intracellular targeting devoid of off-target effects [10]. To overcome these barriers, the delivery vehicle and its constitutive building blocks must meet structural and functional criteria, including biocompatibility, controlled size and surface properties, and reproducible functionalization. For instance, among the aforementioned biological barriers, poor penetration into tumor tissue is a major obstacle for the in vivo efficacy of siRNA or nanocarrier-siRNA therapeutics. Once the nanocarriers have crossed the vascular barrier, they need to navigate through the tumor microenvironment to reach the target cells. The physicochemical properties of nanocarriers play a key role in determining this transport process into the tumor microenvironment [14], and sufficient penetration and distribution of siRNA therapeutics throughout the tumor tissue is vital to produce a therapeutic effect. Nevertheless, siRNA delivery systems are commonly tested in vitro using monolayer cultures where tissue penetration is absent. Cells grown in 2D lack many characteristics of those found in tumors, including gradient of nutrients, a complex microenvironment, and an altered genetic profile 
$[15,16]$. Conversely, multicellular tumor spheroids (MCTS) are 3D cell cultures that resemble several in vivo features of tumors such as high cell density and cell-to-cell contacts, hypoxia, elevated interstitial pressure, resistance to drug treatment, and production of the extracellular matrix (ECM) [15,16].

Cell cultures grown in 3D have been used as an intermediate between monolayer cultures and in vivo studies for the screening of low-molecular weight drugs [17]. The delivery of biomacromolecular drugs, such as siRNA, in MCTS models has not been extensively investigated. Oishi et al. [18] used human hepatocarcinoma spheroids as an in vitro model mimicking the in vivo biology of tumors. They reported that siRNA-loaded lactosylated PEGylated polyplexes could penetrate the tumor spheroids, exerting therapeutic effects through RNA interference. Recent studies have shown that when used in MCTS, oligonucleotide transfection agents, such as cationic lipid complexes, or polyplexes, such as polyethylenimine (PEI) and polyamidoamine (PAMAM) dendrimers, display significantly reduced delivery efficiency in A375, HeLa, and malignant glioma spheroids compared with their performance in 2D cell cultures [19,20]. The limited ability of these complexes to penetrate the 3D multicellular matrix and the ECM protein network is likely due to the large size of the nanocarriers $(\sim 300-900 \mathrm{~nm})$ and their positive surface charge [21]. Other studies have reported the use of prostate cancer spheroid models of LNCaP cells to investigate the ability of $54 \mathrm{~nm}$ poly(E-caprolactone) [22] and $100 \mathrm{~nm}$ poly(methyl methacrylate) [23] nanoparticles loaded with doxorubicin and curcumin, respectively, to diffuse inside the spheroids core. Another study showed limited penetration of liposomes [24] with different diameters $(1 \mu \mathrm{m}$ and $50-150 \mathrm{~nm}$ ) into the LNCaP spheroids, regardless of particle size. It is worth noting that gene knockdown efficacy of any siRNA-delivery system has not been reported for prostate cancer MTSC model to our best knowledge.

Herein, we explore the use of glycogen nanoparticles as a scaffold for engineering siRNA delivery systems and tested these on multicellular prostate tumor spheroids. Glycogen is a randomly branched, tree-like-structured polysaccharide composed of repeating units of glucose connected by linear $\alpha$-D-(1-4) glycosidic linkages with $\alpha$-D-(1-6) branching, which may be isolated from various animal tissues or plants. The roughly spherical glycogen nanoparticles are about $20-150 \mathrm{~nm}$ in diameter depending on the source [25,26], with a polymer-brush-like surface composed of non-reducing ends of glucose [25-28]. Exogenous glycogen is emerging as a bio-nanoparticle for therapeutic applications, as it can be produced with high reproducibility on a large scale, with low cost and ease of modification. A safety evaluation of orally administered glycogen in rats indicated a lack of toxicological and mutagenic response [29]. Glycogen has been modified with hydrophobic octenyl succinate moieties and gadolinium, which enabled the delivery of antigen vaccines [30,31] and MRI imaging probes, respectively [32]. The cationic modification of glycogen using quaternary and tertiary amines to complex and deliver plasmid DNA and siRNA to mammalian cells has been recently reported [29,33,34]. However, in those studies, glycogen sourced from oyster $[33,34]$, which is generally used to induce inflammation in rats, was used [35,36]. In addition, the gene silencing effect was observed with highly positive charged complexes $(+30-40 \mathrm{mV})$, 
which are not optimal for in vivo applications. To prolong in vivo circulation, minimize the immune response, and promote tumor penetration of nanoparticles, neutral or slightly negatively charged surfaces are preferable [14]. In addition, the effect of serum proteins on the integrity and structural changes of glycogen-nucleic acids complexes, the extracellular and intracellular trafficking of the functionalized glycogen in 2D and 3D cell culture, and the in vivo biodistribution were not investigated [29,33,34].

We recently reported the synthesis of lactosylated glycogen nanoparticles that bear surface galactose moieties that exhibit strong, multivalent interactions with lectins overexpressed on prostate cancer cells [37]. Herein, we report a comprehensive and fundamental study to provide a guideline in the bio-engineering of glycogen-nucleic acids constructs with tailored structural features to exhibit a long in vivo circulation life, penetrate multicellular tumor spheroids, and exert a significant gene silencing effect. The advantages of soft glycogen nanoparticles over other polyplexes based on synthetic and natural polymers are their tunable size, degradability, and lack of toxicity at high concentrations. Our results highlight the potential of glycogen nanoparticles for the therapeutic delivery of nucleic acids to the tumor microenvironment and the importance of screening new siRNA-nanocarrier candidates in 3D cell cultures.

\section{Materials and Methods}

\subsection{Materials}

Glycogen from bovine liver, rabbit liver and oyster, sodium cyanoborohydride, ethylenediamine, $\alpha$-amylase from Aspergillus oryzae, Dulbecco's phosphate-buffered saline (D-PBS), trifluoroacetic acid (TFA), 3-(4,5-dimethylthiazol-2-yl)-2,5-diphenyltetrazolium bromide (MTT), human serum, bovine serum albumin, heparin, and RIPA buffer were purchased from Sigma-Aldrich (St. Louis, MO, USA). Methoxy-poly(ethylene)glycol-amine (2 kDa) was obtained from JenKem Technology (Plano, USA). Hydrochloric acid ( $\mathrm{HCl})$, sodium chloride $(\mathrm{NaCl})$, Triton X-100 and Tween-20 were purchased from Chem-Supply (St. Gillman, Australia). Steady-Glo Luciferase Assay System was purchased from Promega (Auburn, Australia). Dialysis tubing (10 kDa molecular weight cutoff) and Protease Inhibitor Cocktail were purchased from Thermo Fisher Scientific (Scoresby, Australia). Alexa Fluor NHS dyes (AF488, AF555, and AF647), Lipofectamine RNAiMAX, SYBr Gold nucleic acid gel stain, Opti-MEM reduced serum medium, AccuMax, trypsin were purchased from Life Technologies (Scoresby, Australia). Dulbecco's Modified Eagle's medium (DMEM) was purchased from Lonza (Allendale, USA). Fetal bovine serum (FBS) was purchased from Bovogen (Keilor East, Australia). Illustra NAP-10 columns were purchased from GE Healthcare and Life Sciences (Silverwater, Australia). Polyvinylidene fluoride (PVDF) membrane was obtained from Pall Corporation (Cheltenham, Australia). PC3 human prostate epithelial cancer cells (CRL-1435), 293T-luc human kidney epithelial cells (CRL-11268), A549 human lung epithelial cancer cells (CCL-185), MDA-MB-231 human breast epithelial cancer cells (HTB-26), and Raw 264.7 murine macrophage cell line (TIB-71) were obtained from ATCC. SiRNA Luc sequence: GL2 duplex (sense: 5'-CGU ACG CGG AAU ACU 
UCG A[dTdT]-3'; anti-sense: 5'-UCG AAG UAU UCC GCG UAC G[dTdT]-3') was purchased from Dharmacon (Lafayette, USA). SiRNA surv sequence: targeting BIRC5 (sense: 5'-GGA CCA CCG CAU CUC UAC A[dTdT]-3'; anti-sense: 5'-UGU AGA GAU GCG GUG GUC C[dTdT]-3') was purchased from Qiagen (Hilden, Germany). SiRNA scr sequence: eGFP Silencer (sense: 5'-GCA CCA UCU UCU UCA AGG A[dTdT]-3'; antisense: 5'-UCC UUG AAG AAG AUG GUG C[dTdT]-3') was purchased from Thermo Fisher Scientific (Scoresby, Australia). Rabbit anti- $\beta$-Actin and rabbit anti-Rab7 monoclonal antibodies were purchased from Cell Signaling Technology (Danvers, United States). Rabbit anti-survivin polyclonal antibody was obtained from Abcam (Cambridge, UK). Mouse antiEEA-1 monoclonal antibody was purchased from BD Biosciences (San Diego, USA). Goat anti-mouse IgG secondary antibody, goat anti-rabbit IgG secondary antibody, and Alexa Fluor conjugates were supplied by Invitrogen (Carlsbad, USA). All chemicals were used as received without further purification. High-purity water with a resistivity greater than 18.2 $\mathrm{M} \Omega \mathrm{cm}^{-1}$ was obtained from a three-stage Millipore Milli-Q plus 185 purification system (Millipore Corporation, Billerica, MA).

\subsection{Synthesis of Glycogen-Ethylenediamine Nanoparticles (NPs)}

Glycogen (100 mg, corresponding to $0.6 \mathrm{mmol}$ of glucose monomers) from bovine liver (bovine glycogen - BG), rabbit liver (rabbit glycogen - RG), or oyster (oyster glycogen -

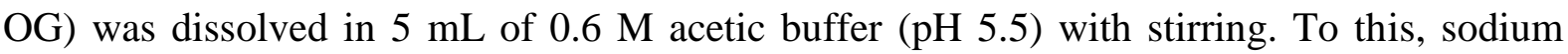
periodate $(26 \mathrm{mg}, 0.12 \mathrm{mmol}$ ) was added and reacted in the dark. After $2 \mathrm{~h}, 36 \mathrm{mg}$ of ethylenediamine (EDA; $0.6 \mathrm{mmol}$ ) was added followed by 10 eq. of sodium cyanoborohydride to $76 \mathrm{mg}$ of sodium periodate $(1.2 \mathrm{mmol})$, and the mixture was stirred overnight. Product was purified by dialysis (tube size $10 \mathrm{kDa}$ ) against Milli-Q water for 3 days (6 times changed) and freeze dried. Yield: $80 \%$. Degree of substitution of modified glycogen was determined by NMR. BG-EDA: ${ }^{1} \mathrm{H}-\mathrm{NMR}\left(400 \mathrm{MHz}, \mathrm{D}_{2} \mathrm{O}, 78{ }^{\circ} \mathrm{C}\right) \delta(\mathrm{ppm})$ : 5.316 (s, 0.8H, H1-4), 5.11 (s, 0.05H, H1-4'), 4.99 (s, 0.1H, H1-6'), 4.91 (s, 0.04H. H1-6), 4.16-3.44 (m, 11H, H2, H3, H4', H5), 3.38 (t, $J=8 \mathrm{~Hz}, 0.1 \mathrm{H}, \mathrm{H} 4), 3.26-2.41\left(\mathrm{~m}, 1.65 \mathrm{H}, \mathrm{H}_{\mathrm{A}}\right.$, $\left.\mathrm{H}_{\mathrm{B}}\right)$. OG-EDA: ${ }^{1} \mathrm{H}-\mathrm{NMR}\left(400 \mathrm{MHz}, \mathrm{D}_{2} \mathrm{O}, 78{ }^{\circ} \mathrm{C}\right) \delta(\mathrm{ppm}): 5.36(\mathrm{~s}, 0.85 \mathrm{H}, \mathrm{H} 1-4), 5.29(\mathrm{~s}$, 0.03H, H1-4'), 5.06 (s, 0.1H, H1-6'), 4.98 (s, 0.02H. H1-6), 4.15-3.5 (m, 7H, H2, H3, H4', H5), 3.43 (t, $J=8 \mathrm{~Hz}, 0.1 \mathrm{H}, \mathrm{H} 4), 3.26-2.7$ (m, 1.2H, HA, $\left.\mathrm{H}_{\mathrm{B}}\right)$. RG-EDA: ${ }^{1} \mathrm{H}-\mathrm{NMR}$ (400 $\left.\mathrm{MHz}, \mathrm{D}_{2} \mathrm{O}, 78{ }^{\circ} \mathrm{C}\right) \delta(\mathrm{ppm}): 5.3(\mathrm{~s}, 0.7 \mathrm{H}, \mathrm{H} 1-4), 5.26$ (s, 0.02H, H1-4'), 5.04 (s, 0.2H, H1-6'), 4.97 (s, 0.01H, H1-6), 4.15-3.5 (m, 6H, H2, H3, H4', H5), 3.43 (s, 0.2H, H4), 3.29-2.7 (m, $\left.1.5 \mathrm{H}, \mathrm{H}_{\mathrm{A}}, \mathrm{H}_{\mathrm{B}}\right)$.

\subsection{Functionalization of Glycogen NPs with Polyethylene Glycol Chains}

Bovine glycogen (BG) was modified with polyethylene glycol (PEG) by a similar reductive amination procedure. Glycogen (100 $\mathrm{mg}, 0.6 \mathrm{mmol}$ of glucose monomers) was dissolved in 5 $\mathrm{mL}$ of $0.6 \mathrm{M}$ acetic buffer, to which $3.21 \mathrm{mg}$ of sodium periodate $(0.015 \mathrm{mmol})$ was added and reacted in the dark. After $2 \mathrm{~h}, 70 \mathrm{mg}$ of methoxy-PEG-amine ( $2 \mathrm{kDa}, 0.035 \mathrm{mmol})$ was added together with an excess of sodium cyanoborohydride $(9.6 \mathrm{mg}, 0.15 \mathrm{mmol})$, and the reaction was left stirring overnight followed by purification by precipitation in cold ethanol 
and isolated by centrifugation (7000 $\mathrm{g}, 10 \mathrm{~min}$ ). The resulting powder was further dissolved in acetic buffer and modified with EDA as described above. Functionalized BG-EDA-PEG was further analyzed by NMR. Yield: $70 \%$. ${ }^{1} \mathrm{H}-\mathrm{NMR}\left(400 \mathrm{MHz}, \mathrm{D}_{2} \mathrm{O}, 78{ }^{\circ} \mathrm{C}\right) \delta(\mathrm{ppm}): 5.35$ (s, 0.8H, H1-4), 5.16 (s, 0.1H, H1-4'), 5.03 (s, 0.03H, H1-6'), 4.95 (s, 0.08H. H1-6), 4.10$3.50\left(\mathrm{~m}, 6.4 \mathrm{H}, \mathrm{H} 2, \mathrm{H} 3, \mathrm{H} 4\right.$ ', H5), $3.41(\mathrm{t}, J=8 \mathrm{~Hz}, 0.12 \mathrm{H}, \mathrm{H} 4), 3.21-2.77\left(\mathrm{~m}, 0.5 \mathrm{H}, \mathrm{H}_{\mathrm{A}}, \mathrm{H}_{\mathrm{B}}\right)$, $2.21\left(\mathrm{~s}, 6.5 \mathrm{H}, \mathrm{H}_{\mathrm{c}}\right), 1.89\left(\mathrm{~s}, 0.07 \mathrm{H}, \mathrm{H}_{\mathrm{d}}\right)$.

\subsection{Labeling of BG-EDA and BG-EDA-PEG with Alexa Fluor-NHS Dyes}

Glycogen-EDA (10 mg) was dissolved in $2 \mathrm{~mL}$ of $0.1 \mathrm{M}$ sodium bicarbonate buffer at $\mathrm{pH} 8$ and mixed with $100 \mu \mathrm{L}$ of $1 \mathrm{mg} \mathrm{mL}^{-1}$ Alexa Fluor-NHS dye (either Alexa Fluor-488 or Alexa Fluor-555) and kept stirring overnight in the dark. Excess dye was removed by purification on a NAP-10 filter column and freeze dried.

\subsection{Dynamic Light Scattering (DLS), ל-potential and Molecular Weight (MW) Measurements}

The hydrodynamic diameter, $\zeta$-potential, and molecular weights of the particles were measured using a Zetasizer Nano-ZS (Malvern Instruments, Malvern, UK) equipped with He$\mathrm{Ne}$ ion laser $(\lambda=633 \mathrm{~nm})$. For the DLS measurement, $50 \mu \mathrm{L}$ of particle solution $\left(3 \mathrm{mg} \mathrm{mL}^{-1}\right)$ in Milli-Q water was analyzed in a micro cuvette (ZEN0040, Malvern Instruments). The measurements were performed using a standard operation procedure with automatic attenuation and measurement position. For the $\zeta$-potential measurements, $750 \mu \mathrm{L}$ of particle solution (3 $\left.\mathrm{mg} \mathrm{mL}^{-1}\right)$ in Milli-Q water was introduced into a folded capillary cell (DTS1070, Malvern Instruments) and measurements were performed in a similar manner. For molecular the weight measurements, a static light scattering method was used, testing glycogen concentrations ranging from 0.1 to $2 \mathrm{mg} \mathrm{mL}^{-1}$. Toluene was used as a standard. The molecular weights were obtained from the resulting Debye plot of scattering intensity against concentration.

\subsection{Glycogen-siRNA Construct Formation and Characterization}

Complex formation of modified glycogen with siRNA was monitored using $10 \%$ Tris-borate EDTA TBE polyacrylamide gel electrophoresis. Samples were prepared as follows: 15 pmol of luciferase silencer siRNA was mixed with cationic glycogen at different weight ratios (w/w 1-7) in $30 \mu \mathrm{L}$ of $30 \mathrm{mM}$ phosphate-buffered saline (PBS) buffer. The prepared samples were allowed to equilibrate for $10 \mathrm{~min}$, and $5 \mu \mathrm{L}$ of nucleic acid loading buffer was added. Then, $25 \mu \mathrm{L}$ of each sample was loaded onto the gel and ran in an electrophoresis chamber for $1 \mathrm{~h}$ at $150 \mathrm{~V}$. siRNA retardation was evaluated by staining the gel with SyBr Gold reagent and imaged using a ChemiDoc XRS+ Imaging System (BioRad, USA). The size and charge of the glycogen constructs at different ratios were further evaluated with DLS. To estimate the number of siRNA molecules within a single construct, we approximated each glycogensiRNA construct as a large sphere that is filled with smaller spheres at a defined packing density $\sigma$. Each smaller sphere is assumed to be coated with siRNA molecules, where we conservatively approximate each siRNA molecule to occupy a circular surface area on each 
BG-EDA particle. We allow each siRNA molecule that is adsorbed on a BG-EDA particle to exclude a volume equivalent of a sphere of diameter equivalent to the maximal dimension of a siRNA molecule. The number of siRNA per glycogen-siRNA construct, $N_{\text {siRnA }} / N_{\text {construct }}$, may therefore be approximated by:

$$
\frac{N_{\text {siRNA }}}{N_{\text {construct }}} \approx \frac{4 \sigma r_{\text {construct }}^{3} r_{B G-E D A}^{2}}{r_{\text {siRNA }}^{2}\left(r_{B G-E D A}+2 r_{\text {siRNA }}\right)^{3}}
$$

where $r_{\text {construct }}, r_{\mathrm{BG}-\mathrm{EDA}}$, and $r_{\mathrm{siRNA}}$ indicate the radii of the glycogen-siRNA construct, BGEDA, and the siRNA, respectively. We set $r_{\text {construct }}$ and $r_{\text {BG-EDA }}$ or $r_{\text {BG-EDA-PEG }}$ to $168 \mathrm{~nm}$ and $24 \mathrm{~nm}$, respectively, as per data in Table S1 in Supporting Information. We allow the $r_{\text {siRNA }}$ to vary between 5 and $7 \mathrm{~nm}$ and $\sigma$ to vary between 0.60 and 0.74 , which indicates loose random packing and dense regular packing of spheres, respectively.

\subsection{Small-Angle X-Ray Scattering}

Small-angle X-ray scattering (SAXS) was performed at the Australian Synchrotron, using a $3.3 \mathrm{~m}$ camera setup, detected with a $1 \mathrm{M}$ Pilatus detector. Particles were dissolved in $1 \mathrm{~mL}$ of Milli-Q water, briefly sonicated, then filtered with an Aerodisc $0.22 \mu \mathrm{m}$ syringe filter and dispersed into a 96 -well plate $(100 \mu \mathrm{L})$. SAXS patterns were collected at $25{ }^{\circ} \mathrm{C}$. Data were analyzed using the PRIMUS software [38], where Guinier fits were performed to extract the $R_{\text {g. }}$

\subsection{Atomic Force Microscopy}

Atomic force microscopy (AFM) measurements were performed with a MFP-3D Asylum research instrument, with Bruker RTESPA-300 cantilevers in tapping mode. Both particles types where dispersed onto a silicon wafer at a concentration of $0.01 \mathrm{mg} \mathrm{mL}^{-1}$. The silicon wafer was first sonicated in ethanol, then washed with detergent and Milli-Q water, and then plasma-cleaned and dried under a stream of nitrogen.

\subsection{In vivo Biodistribution Imaging}

Fluorescent BG-EDA CY7.5 or BG-EDA-PEG CY7.5-siRNA constructs (100 $\mu \mathrm{L}$ with concentration of $1 \mathrm{mg} \mathrm{mL}^{-1}$ ) were prepared in PBS solution for in vivo imaging. Animal experiments were conducted under the guidelines approved by the Animal Care and Ethics Committee in Xiamen University (Protocol number: XMULAC20160020). BALB/c mice of 7-12 weeks of age were maintained in animal facilities with feeding of a standard pellet diet and water ad libitum. A $100 \mu \mathrm{L}$ of sample dissolved in PBS was injected intravenously into a mice model via tail vein at a concentration of $1 \mathrm{mg} \mathrm{mL}^{-1}$. For constructs with scramble siRNA, $5 \mu \mathrm{L}$ of sample $\left(1 \mathrm{mg} \mathrm{mL}^{-1}\right)$ was gently mixed with $5 \mu \mathrm{L}$ of scramble siRNA solution $(20 \mu \mathrm{M})$ before dilution in $200 \mu \mathrm{L}$ PBS at room temperature for $30 \mathrm{~min}$ before injection. At different time intervals, an In Vivo Imaging System IVIS ® Lumina II (Caliper Life Sciences, Hopkinton, MA) was used to collect the fluorescence images; $745 \mathrm{~nm}$ as an excitation filter and $810-875 \mathrm{~nm}$ as an emission filter were used for collecting the Cy7.5 (maximum 
excitation at $788 \mathrm{~nm}$ and maximum emission at $808 \mathrm{~nm}$ ) fluorescence signals. Radiation efficiency was collected and presented in form of average \pm standard deviations for 3 repeated experiments. After $24 \mathrm{~h}$, mice were sacrificed and major organs (including heart, liver, spleen, lung, kidney, and brain) isolated for ex vivo fluorescence imaging to determine the tissue distribution of glycogen-siRNA constructs.

\subsection{Analysis of Liver Toxicity}

BG-EDA $(5 \mu \mathrm{L})$ and BG-EDA-PEG $\left(1 \mathrm{mg} \mathrm{mL}^{-1}\right)$ were mixed with scramble DNA solution (5 $\mathrm{mL}, 20 \mu \mathrm{M})$ and incubated for $30 \mathrm{~min}$. After diluting in sterile phosphate-buffered solution $(200 \mu \mathrm{L})$, the mixed solution $(100 \mu \mathrm{L})$ was injected via tail vein. PBS was injected intravenously as a control. As the fluorescence signal in the liver at around $8 \mathrm{~h}$ after intravenous injection happened to be the strongest, liver cytotoxicity was evaluated $8 \mathrm{~h}$ after injection. To prove that BG-EDA and BG-EDA-PEG do no harm the liver after injection, liver cytotoxicity was also evaluated $24 \mathrm{~h}$ after intravenous injection.

\subsubsection{Biochemical Analysis}

Blood was collected $8 \mathrm{~h}$ and $24 \mathrm{~h}$ after intravenously injection. After $10 \mathrm{~min}$ of centrifugation at $3000 \mathrm{rpm}$, serum was collected for the detection of liver-related enzymes such as alanine aminotransferase (ALT) and aspartate aminotransferase (AST). ALT and AST were detected by ALT and AST detection assay kits, respectively, according to the protocols supplied by the manufacturer (Sigma Aldrich).

\subsubsection{Histology and Immunohistochemistry Evaluation}

Mice were sacrificed $8 \mathrm{~h}$ and $24 \mathrm{~h}$ after intravenous injection and livers were excised. After dehydration by $15 \%$ and $30 \%$ sucrose, livers were embedded in optimal cutting temperature compound and sliced into sections with a thickness of $5 \mu \mathrm{m}$. Hematoxylin \& eosin (H\&E) staining was applied for histology and immunohistochemistry evaluation.

\subsection{Degradation of Glycogen NPs by $\alpha$-Amylase}

The rate of glycogen particle degradation by $\alpha$-amylase was determined using a phenolsulfuric acid assay [39]. For the assay, $200 \mu \mathrm{L}$ of $0.7 \mathrm{mg} \mathrm{ml}^{-1}$ solution of BG, BG-EDA, or BG-EDA-PEG were treated with $\alpha$-amylase solution in PBS pH $7.4\left(0.5 \mathrm{U} \mathrm{mg}^{-1}\right)$ for $3 \mathrm{~h}$, followed by separation of particles from enzyme and undigested glycogen using spin columns with pore size of $30 \mathrm{kDa}$. The filtrate was collected and divided into $50 \mu \mathrm{L}$ samples. Then, $150 \mu \mathrm{L}$ of $98 \%$ concentrated sulfuric acid was added to each sample followed by immediate addition of $30 \mu \mathrm{L}$ of $5 \%$ phenol solution, and the samples were incubated for $5 \mathrm{~min}$ at $90{ }^{\circ} \mathrm{C}$. The UV-Vis absorbance was measured at $490 \mathrm{~nm}$ by an Infinite M200 microplate reader (Tecan, Switzerland) using a 96-well plate (Costar 3596, Corning, MA, USA). For total glucose assay, particles were treated with $200 \mu \mathrm{L}$ of $0.2 \mathrm{M}$ TFA solution for $3 \mathrm{~h}$ at $80{ }^{\circ} \mathrm{C}$, instead of $\alpha$-amylase, followed by the procedure described above.

\subsection{Determination of pKa of Particles}


The pKa of amine-functionalized glycogen particles was measured by potentiometric titration. For the measurement, $10 \mathrm{mg}$ of BG and BG-EDA was dissolved in $5 \mathrm{~mL}$ of $200 \mathrm{mM}$ $\mathrm{NaCl}$ solution, and $\mathrm{pH}$ was adjusted to 11 using $0.1 \mathrm{~N} \mathrm{NaOH}$. The suspension was titrated with $0.01 \mathrm{~N} \mathrm{HCl}$ to $\mathrm{pH}$ of $\sim 2.5$. The $\mathrm{pKa}$ of the particles was determined using the first derivative method.

\subsection{Glycogen-siRNA Construct Stability in Bovine Serum}

Glycogen-siRNA constructs at different ratios were incubated with 20\% FBS for $24 \mathrm{~h}$ at 37 ${ }^{\circ} \mathrm{C}$. For heparin samples, loaded siRNA was replaced from glycogen construct with excess of heparin after pre-incubation with serum proteins. Samples were mixed with nucleic acid loading buffer, and 10\% TBE polyacrylamide gel electrophoresis was performed.

\subsection{Nanoparticle Tracking Analysis (NTA)}

Glycogen particles labeled with Alexa Fluor-488 were assembled into glycogen-siRNA constructs at $\mathrm{w} / \mathrm{w}$ ratios of 4:1 and 6:1 for BG-EDA and BG-EDA-PEG, respectively. The size of the resulting particles was analyzed in PBS at a concentration of $1 \mu \mathrm{g} \mathrm{mL}^{-1}$ using a NanoSight NS300 (Malvern Instruments, Malvern, UK) by fluorophore excitation with a 488 $\mathrm{nm}$ laser and tracking with a sCMOS camera.

\subsection{Stochastic Optical Reconstruction Microscopy (STORM)}

For STORM analysis, BG-EDA and BG-EDA-PEG were assembled into constructs with Alexa Fluor 647 conjugated to siRNA. Glycogen-siRNA constructs were added to DMEM medium supplemented with $10 \%$ FBS to a final glycogen concentration of $5 \mu \mathrm{g} \mathrm{mL}-1$ and incubated in $37{ }^{\circ} \mathrm{C}$ for $5 \mathrm{~h}$. STORM images were acquired using a Nikon N-STORM system configured to achieve total internal reflection fluorescence imaging (TIRF). The perfect focus system and TIRF angle were adjusted and tuned to maximize the signal-to-noise ratio. The fluorophore was excited by $647 \mathrm{~nm}$ laser line. No activation by ultraviolet light was used. Fluorescence was collected by means of a Nikon $100 \times 1.4$ NA oil immersion objective and passed through a quad-band pass dichroic filter. All time-lapses were recorded onto a $256 \times$ 256 pixel region by an EMCCD camera. For each channel, 3000-5000 frames were sequentially acquired. STORM movies were analyzed with the STORM module of the NIS Elements Nikon software.

\subsection{Cell Viability}

Cell viability was measured using standard MTT assay protocol. Cells (PC3, A549, Raw 647.5, MDA-MB-231, 293T) were plated on 96-well plates (Costar 3596, Corning, MA, USA) with seeding density of $1 \times 10^{4}$ cells per well in $100 \mu \mathrm{L}$ of DMEM medium supplemented with $10 \%$ fetal bovine serum. On the next day, glycogen particles were added to the culture media and were incubated with cells for $24 \mathrm{~h}$, after which MTT reagent was added. After $4 \mathrm{~h}$, the resulted formazan crystals were dissolved in dimethyl sulfoxide, and the well absorbance was measured at $554 \mathrm{~nm}$ and $670 \mathrm{~nm}$ (as reference) with an Infinite M200 microplate reader (Tecan, Switzerland). 


\subsection{Laser Scanning Confocal Microscope Imaging}

PC3 and 293T cells were seeded at 40,000 cells per well in Labtek 8-well chamber slides and incubated overnight. The medium was replaced with fresh standard media. BG-EDA-siRNA (w/w 4:1) and BG-EDA-PEG-siRNA (w/w 6:1) labeled with Alexa Fluor-555 were added to a final concentration of $100 \mathrm{nM}$ of siRNA in serum-free DMEM. After $4 \mathrm{~h}$ of incubation, the medium was removed. Cells were washed three times with pre-warmed PBS-BSA (1\% BSA in PBS) to remove unbounded particles from cells and to block non-specific binding on the cell surface. Cells were fixed with $4 \%$ paraformaldehyde for $15 \mathrm{~min}$, washed, permeabilized with $0.1 \%$ TritonX-100 solution in PBS for $5 \mathrm{~min}$, and washed again three times with $1 \%$ BSA in PBS. Samples were then incubated for $1.5 \mathrm{~h}$ with mouse anti-EEA1 monoclonal antibody or rabbit anti-Rab7 monoclonal antibody at $2.5 \mu \mathrm{g} \mathrm{mL} \mathrm{m}^{-1}$. Cells were washed three times and incubated for $1 \mathrm{~h}$ with goat anti-mouse or goat anti-rabbit Alexa Fluor 647 conjugate antibody $\left(2 \mu \mathrm{g} \mathrm{mL} \mathrm{mL}^{-1}\right)$. Cells were imaged with a Nikon A1R confocal microscope with a $60 \times 1.4$ NA oil immersion objective. Pearson's correlation coefficients were obtained with NIS-Elements analysis software.

\subsection{In vitro Gene Knockdown}

Luciferase-expressing 293T human kidney epithelial cells were plated on 96-well white plates (Costar 3917, Corning, MA, USA) with a seeding density of $1 \times 10^{4}$ cells per well in $100 \mu \mathrm{L}$ of DMEM medium supplemented with $10 \%$ fetal bovine serum and incubated at 37 ${ }^{\circ} \mathrm{C}$, in $5 \% \mathrm{CO}_{2}$ for $24 \mathrm{~h}$. Glycogen-siRNA constructs were prepared according to the procedure described above at w/w 4:1 and w/w 6:1 for BG-EDA and BG-EDA-PEG, respectively, and incubated with cells at $100 \mathrm{nM}$ of siRNA concentration in serum-free DMEM media. After $2 \mathrm{~h}$, complete growth medium containing 10\% FBS was added. Lipofectamine RNAiMax transfection agent (Life Technologies) was used as a control. Gene knockdown was evaluated after $48 \mathrm{~h}$ and $72 \mathrm{~h}$ of incubation using Steady-Glo Luciferase kit (Promega) following the manufacturer's protocol, and reading the luminescence using an Infinite M200 microplate reader (Tecan, Switzerland). PC3 cells were cultured under the same condition described for 293TLuc cells on 12-well plates (Costar, Corning, MA, USA) with seeding density of $8.0 \times 10^{4}$ cells per well. Gene knockdown was evaluated using flow cytometry. Briefly, cells were washed with D-PBS, detached, fixed with $4 \%$ paraformaldehyde and permeabilized using 0.1\% TritonX-100 and 1\% BSA in PBS solution. Survivin knockdown was investigated using rabbit anti-survivin polyclonal antibody (Abcam). A 1:200 dilution in $200 \mu \mathrm{L}$ was used for both antibodies with $2 \mathrm{~h}$ of incubation time. Goat anti-rabbit Alexa Fluor 647 conjugate antibody $\left(2 \mu \mathrm{g} \mathrm{mL} \mathrm{m}^{-1}\right)$ was used for secondary staining. Cells were always washed twice with $1 \%$ BSA in D-PBS and centrifuged at $400 \mathrm{rpm}$ between each step. Cells were analyzed using a BD Accuri ${ }^{\mathrm{TM}}$ C6 flow cytometer; 10,000 cells were collected per treatment. Data were analyzed using FlowJo.

\subsection{Western Immunoblotting}

PC3 cells were transfected with $100 \mathrm{nM}$ siRNA/BG-EDA and BG-EDA-PEG constructs according to the method above. After $72 \mathrm{~h}$, cells were washed twice in D-PBS, trypsinized, 
and washed two more times in D-PBS, and centrifuged (400 rpm, $5 \mathrm{~min}$ ). Whole cell extract was prepared by lysing cells for $30 \mathrm{~min}$ on ice in RIPA buffer containing a protease inhibitor cocktail and separated from cell debris by centrifugation (16,000 rpm, $\left.15 \mathrm{~min}, 4{ }^{\circ} \mathrm{C}\right)$. Fifty micrograms of protein extract was separated by SDS polyacrylamide gel electrophoresis and transferred to a PVDF membrane using standard protocols. The membrane was washed twice with PBS, followed by incubation with blocking buffer $(5 \% \mathrm{w} / \mathrm{v}$ skim milk powder in PBSTeen20). The membrane was incubated with anti-survivin and anti- $\beta$-Actin primary antibodies (1:5000 in 5\%BSA/PBS-T buffer) overnight. The membrane was then incubated with anti-rabbit peroxidase-conjugated antibody (1:10000 in PBS-T). Bound antibodies were detected using ClarityMax Western ECL Substrate and imaged with a ChemiDoc XRS+ Imaging System (BioRad, USA).

\subsection{D Cell Culture Preparation and Analysis}

293T-Luc cells and PC3 were plated on Ultra-low attachment Spheroid 96-well plates (Coring 4515, USA) with initial seeding density of $1.0 \times 10^{3}$ cells per well in DMEM containing 10\% FBS and spun down at $200 \mathrm{rpm}$ for $2 \mathrm{~min}$. Growth and formation of spheroids were monitored under an inverted microscope with a $10 \times$ objective. Media was replaced every 2 days until cells were mature and compact spheroids were formed (typically 4-5 days for 293T cells and 7-9 days for PC3 cells). For experiments, glycogen-siRNA constructs were prepared as described previously and administered at a siRNA concentration of $100 \mathrm{nM}$. The transfection protocol was identical to that used for the $2 \mathrm{D}$ cell culture. For spheroid penetration analysis, glycogen particles were labeled with Alexa Fluor-555. After 24 $\mathrm{h}$ incubation, spheroids were harvested, washed in D-PBS, and fixed for $1 \mathrm{~h}$ in $4 \%$ paraformaldehyde, and washed again twice with $1 \%$ BSA in D-PBS. To visualize the nucleus, spheroids were stained with Hoechst 33258 at $0.2 \mathrm{mg} \mathrm{mL}^{-1}$. Spheroids were placed in $3 \%$ agarose, dehydrated in an ethanol gradient, and embedded into paraffin blocks. Sections were taken with a microtome with $5 \mu \mathrm{m}$ thicknesses and analyzed using a confocal microscope at 20x and 60x magnifications. For knockdown efficacy, spheroids were dissociated with AccuMax solution for 20 min. Knockdown efficacy in 293T spheroids was determined using the Steady-Glo Luciferase Kit as described by the manufacturer. Knockdown efficacy in PC3 spheroids was evaluated by Western Immunoblotting using cell lysate obtained from 10 spheroids.

\section{Results and Discussion}

\subsection{Tailoring the Physicochemical Properties of Glycogen-siRNA Constructs}

Glycogen nanoparticles from different bio-sources and with different structural features have been synthetically functionalized and characterized [34,40]. First, we sought to investigate the effect of size and branching structure of glycogens sourced from bovine liver (BG), rabbit liver (RG), and oyster (OG) on the physicochemical properties of glycogen constructs with siRNA. The hydrodynamic diameters of glycogen nanoparticles differ depending on the source, from $20 \mathrm{~nm}$ for BG to $80 \mathrm{~nm}$ for RG (see Table 1), as determined by DLS. This is consistent with the respective molecular weight of the source material (see Table 1), 
determined as described in the Materials and Methods. BG has the lowest molecular weight, hence a small size and a narrow size distribution, whereas OG and RG show significantly higher molecular weights, thus larger sizes and broader size distributions. The $\zeta$-potentials of the glycogen nanoparticles range from about $-11 \mathrm{mV}$ to $0 \mathrm{mV}$. This is consistent with a small amount of phosphate present on the particle surfaces, resulting from the action of phosphorylating enzymes in vivo $[41,42]$. The degree of branching was calculated by ${ }^{1} \mathrm{H}$ NMR (Figure S1) using the ratio between the H1-6 proton signal at $\delta=4.93 \mathrm{ppm}$, which is ascribed to the glucose residues linked by $\alpha-1,6$-glycosidic bonds, and the H1-4 proton signal at $\delta=5.32 \mathrm{ppm}$ signal, which arises from the total glucose units within the glycogen structure. Similarly, the degree of reducing chain ends was evaluated using the ratio between the $\mathrm{H} 4$ proton signal observed at $\delta=3.38 \mathrm{ppm}$ and the H1-4 proton signal at $\delta=5.32 \mathrm{ppm}$. The NMR spectra indicate that the RG nanoparticles are the most highly branched with a high inner density.

Table 1. Physicochemical properties of glycogen nanoparticles from bovine liver (BG), rabbit liver $(\mathrm{RG})$ and oyster $(\mathrm{OG})$

\begin{tabular}{|c|c|c|c|c|c|}
\hline Particle & $\begin{array}{c}D_{\mathrm{h}} \\
(\mathrm{nm})\end{array}$ & $\begin{array}{c}\zeta \\
(\mathrm{mV})\end{array}$ & $\begin{array}{c}M_{\mathrm{W}} \\
(\mathrm{kDa})\end{array}$ & $\begin{array}{c}\text { Degree of } \\
\text { branching } \\
(\%)\end{array}$ & $\begin{array}{c}\text { Degree of } \\
\text { chain ends } \\
(\%)\end{array}$ \\
\hline BG & $\begin{array}{c}19 \pm 4 \\
\text { (PDI: } 0.21)\end{array}$ & $-11 \pm 7$ & $360 \pm 25$ & 8.8 & 11.9 \\
\hline $\mathrm{RG}$ & $\begin{array}{c}80 \pm 35 \\
\text { (PDI: 0.16) }\end{array}$ & $-2 \pm 3$ & $8550 \pm 980$ & 15.6 & 21.8 \\
\hline OG & $\begin{array}{c}55 \pm 20 \\
\text { (PDI: 0.14) }\end{array}$ & $0 \pm 4$ & $5190 \pm 355$ & 5.1 & 7.2 \\
\hline $\begin{array}{l}D_{\mathrm{h}}-\text { hydrod } \\
\text { determined } \\
\text { scattering } \\
{ }^{1} \mathrm{H}-\mathrm{NMR} \text { sp }\end{array}$ & $\begin{array}{l}\text { mic diameter } \\
\text { microelectro } \\
\text { [37]; degre } \\
\text { oscopv: PDI }\end{array}$ & $\begin{array}{l}\text { rmined b } \\
\text { esis; } M \\
\text { branchin } \\
\text { vdisners }\end{array}$ & $\begin{array}{l}\text { namic light } \\
\text { nolecular } \\
d \text { degree of } \\
\text { dex. }\end{array}$ & $\begin{array}{l}\text { ring (DLS) } \\
\text { determin } \\
\text { ends wer }\end{array}$ & $\begin{array}{l}\text { zeta potent } \\
\text { y static li } \\
\text { ermined fr }\end{array}$ \\
\hline
\end{tabular}

The cytotoxicity of glycogen nanoparticles from different sources was evaluated by MTT viability assays performed on four different cell lines i.e., human prostate, breast and lung cancer cells (PC3, MDA-MB-231, and A549), and murine macrophages (Raw 264.7) (Figure S2). Glycogen particles did not affect cell viability when incubated at concentrations ranging from 0.015 to $0.5 \mathrm{mg} \mathrm{mL}^{-1}$, indicating that the particles are not cytotoxic.

To engineer glycogen-siRNA constructs, the physicochemical properties of glycogen nanoparticles must be carefully controlled. We incorporated amine moieties onto BG, RG, and $\mathrm{OG}$ glycogens to provide a positive surface charge to the glycogen nanoparticles. Glycogen-siRNA constructs formation is based on the association of negatively charged 
siRNA molecules with glycogen nanoparticles bearing amine groups (Figure 1A). Primary and secondary amine functionalities were introduced by reductive amination [43] using EDA (Figure S3). The degree of substitution (DS) with EDA was evaluated by ${ }^{1} \mathrm{H}-\mathrm{NMR}$ (Figure S4 and Figure S5) from the integration of EDA proton signals at $\delta=3.05 \mathrm{ppm}$ and $\delta=2.88$ ppm. Peaks integration indicated a 40\%, 36\%, and 29\% DS for BG-EDA, RG-EDA, and OGEDA, respectively. DLS and $\zeta$-potential data, shown in Table S1, indicate that the resulting cationic glycogen nanoparticles increased slightly in size and exhibited a $\zeta$-potential of approximately $30-40 \mathrm{mV}$. This functionalization route is effective for introducing cationic moieties without compromising the colloidal stability of glycogen nanoparticles. In addition, BG was modified with methoxy-PEG-amine $(2 \mathrm{kDa})$ using a similar reductive amination reaction. Even a low extent of PEGylation (DS 2.5\%) is expected to improve the biofunctional properties of the glycogen constructs.

The structural changes introduced by PEGylation on glycogen nanoparticles were examined by a comparison between DLS and SAXS measurements. Table S1 shows a negligible change in the hydrodynamic diameter of BG-EDA upon PEGylation, as measured by DLS. In contrast, SAXS data (Figure S6, Table S1) show differences in scattering at low $q$-ranges, where both solvent-subtracted data sets are normalized with respect to $I_{0}$. The diameters of gyration, $D_{\mathrm{g}}$, of BG-EDA, BG-EDA-PEG, RG-EDA, and OG-EDA were found to be 11, 21, 41 , and $45 \mathrm{~nm}$, respectively, using Guinier analysis. The larger diameters obtained for BGEDA, RG-EDA, and OG-EDA by DLS may be attributed to the hydrodynamic shell, which is not taken into account in SAXS measurements. It is known that roughly spherical glycogen nanoparticles have a very well-ordered region of strongly bound water in comparison to linear polysaccharides [44], highlighting the strong influence of chain architecture on the structuring of water. Interestingly, a consistent size value was observed for BG-EDA-PEG using the two different techniques, which suggests that the hydrophilic PEG chains partially disrupt the hydrating water shell of BG-EDA.

The ability of BG-EDA, RG-EDA, and OG-EDA to form constructs with siRNA was studied by polyacrylamide gel electrophoresis (Figure 1B). Naked siRNA was used as a negative control, indicating unbound siRNA, and retardation of migration was examined at increasing glycogen-to-siRNA w/w ratios. For BG-EDA, RG-EDA, and OG-EDA, complete band disappearance was observed at a glycogen-to-siRNA w/w ratio of 5:1, whereas for BG-EDAPEG a higher ratio of 7 was required. Taking into account the molecular weight of glycogen nanoparticles (Table 1), one nanoparticle of BG-EDA or BG-EDA-PEG can bind to approximately 5 molecules of siRNA, whereas RG-EDA and OG-EDA nanoparticles can bind to up to 125 and 74 siRNA molecules, respectively. DLS data (Figure 1C) indicated that the binding of siRNA to RG-EDA and OG-EDA resulted in large aggregates of approximately $300-400 \mathrm{~nm}$ in diameter. This suggests that the larger glycogen nanoparticles sourced from rabbit liver and oyster are not the optimal systems for complexation of nucleic acids if fine control over the size of constructs is required. The uncontrolled and rapid aggregation process is driven by electrostatic interactions between glycogen and siRNA. Conversely, for both BG-EDA and BG-EDA-PEG at w/w ratio of 4:1 and w/w ratio of 6:1 
respectively, constructs sizes were approximately $150 \mathrm{~nm}$ (Figure 1C). A dramatic increase in size from $150 \mathrm{~nm}$ to 800-900 nm was observed when BG-EDA and BG-EDA-PEG were mixed with siRNA at higher w/w ratios of 5:1 and 7:1, respectively. An increase in size at higher ratios is likely due to aggregation induced by free BG-EDA and BG-EDA-PEG nanoparticles, bridging together negatively charged constructs and exposing siRNA molecules on the outer shell. The $\zeta$-potential of BG-EDA-siRNA and BG-EDA-PEG-siRNA constructs increased from $-25 \mathrm{mV}$ at w/w ratio of $1: 1$ to slightly negative values at w/w ratios of 4:1 to 6:1 (Figure 1D).

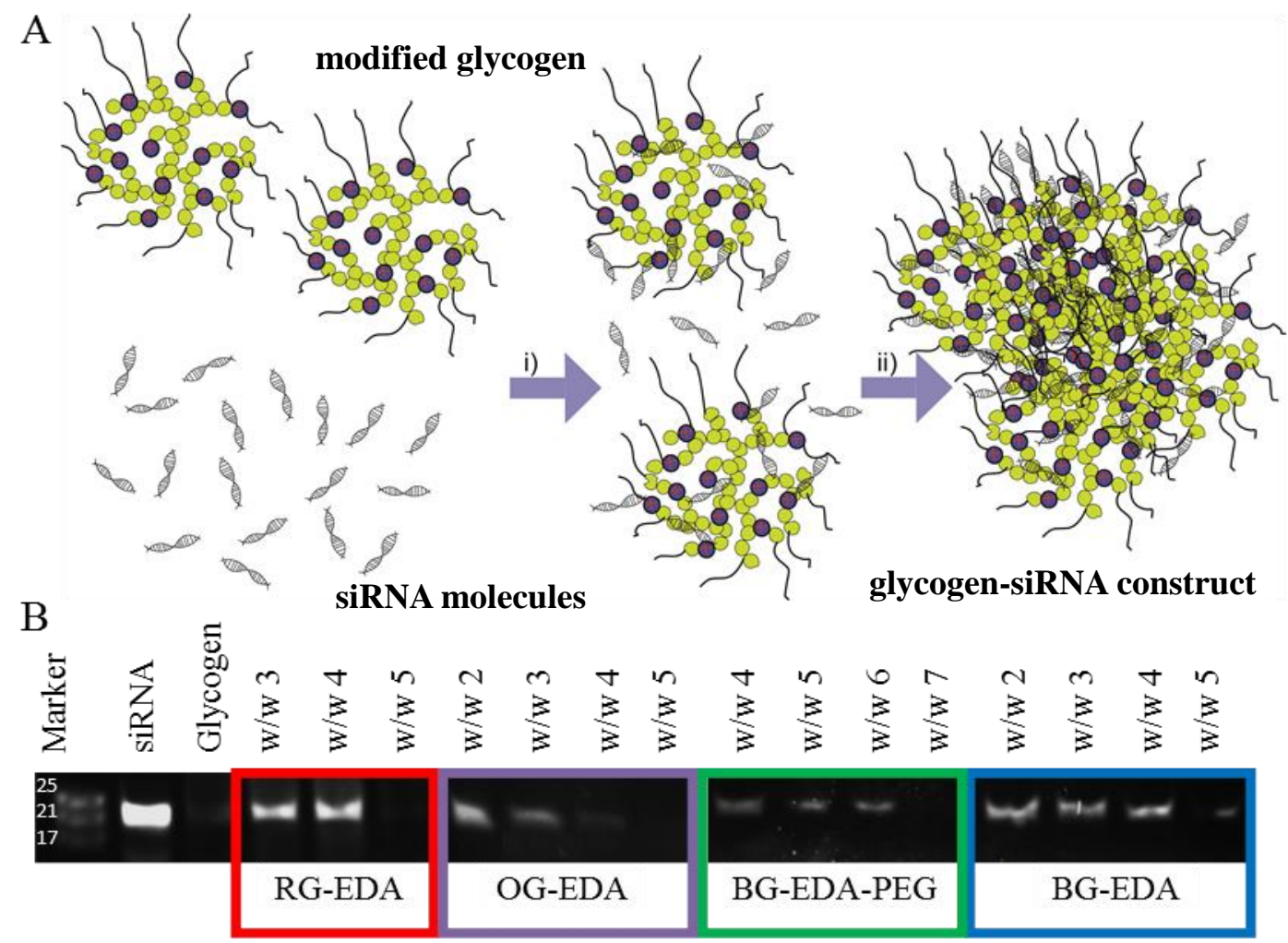

$\mathrm{C}$

$\mathrm{D}$

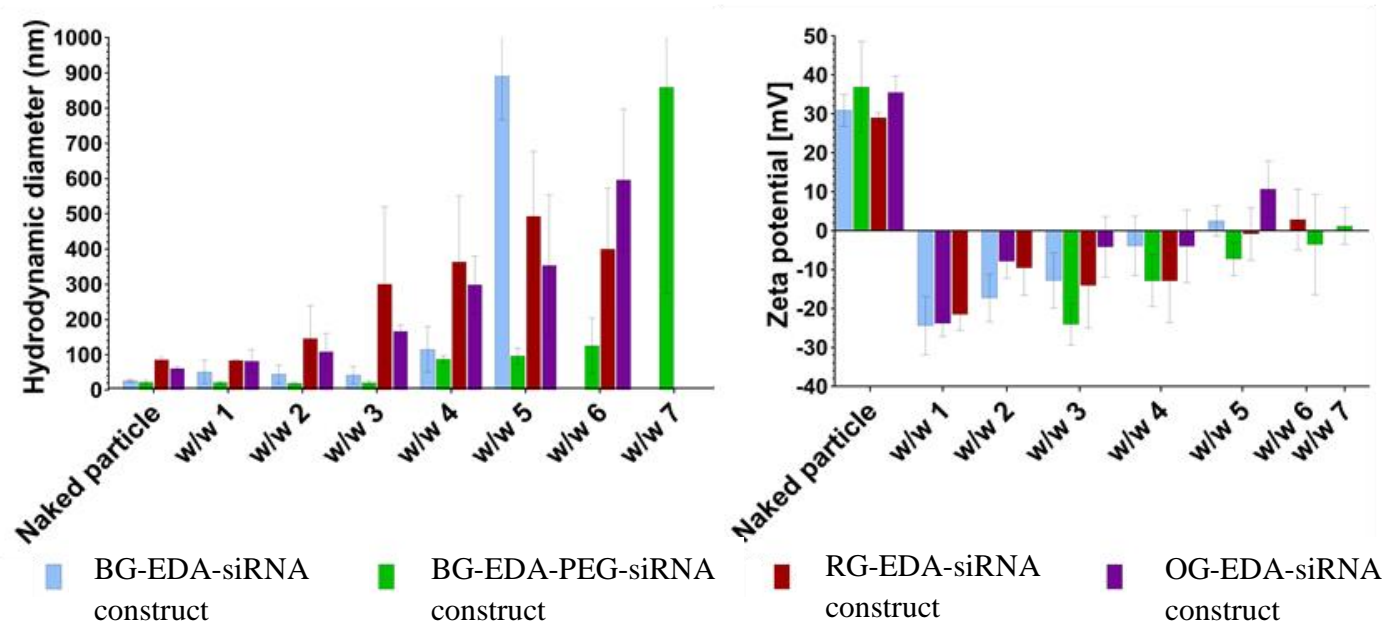


Figure 1. Engineering of glycogen-siRNA constructs with optimal physicochemical properties. A) Schematic of glycogen-siRNA constructs formation: (i) mixing of siRNA with glycogen nanoparticles at the optimized w/w ratio; (ii) formation of glycogen-siRNA construct with controlled size and surface charge. Dark circles in the glycogen structure indicate amine functionalities and black strands represent flexible PEG chains. B) Polyacrylamide gel electrophoresis analysis of constructs formed at different particle-tosiRNA w/w ratios: RG-EDA (red box); OG-EDA (purple box); BG-EDA-PEG (green box); and BG-EDA (blue box). C) Hydrodynamic diameter of BG-EDA-, BG-EDA-PEG-, RGEDA-, and OG-EDA-siRNA constructs at increasing w/w ratios. D) $\zeta$-potential of BG-EDA-, BG-EDA-PEG-, RG-EDA-, and OG-EDA-siRNA constructs at increasing w/w ratios.

The rational design of gene delivery platforms imposes restrictions on the structural features of the carriers where a size ranging from 50 to $150 \mathrm{~nm}$ and a neutral or slightly negative surface charge are optimal parameters [14]. These parameters are crucial for controlling the carrier stability in biological fluids in vivo and their ability to penetrate the extracellular matrix. We therefore selected BG-EDA and BG-EDA-PEG as scaffolds for siRNA complexation, where an optimal size, surface charge, and siRNA complexation were obtained at w/w ratios of $4: 1$ and 6:1, respectively. Though the gel retardation data indicate incomplete complexation at these ratios, unbound negatively charged siRNA molecules are unable to cross cell membranes and are readily degraded in serum [1]. These results suggest that a trade-off between the siRNA loading capacity, the resulting size and surface charge of the complex is of importance. Using conservative scaling arguments (see Materials and Method), we estimated that each construct accommodates between 1200 and 3300 molecules of siRNA (Figure 2A), which is similar to the siRNA loading capacity of other polymeric carriers evaluated in early clinical trials such as CALAA-01 [8].
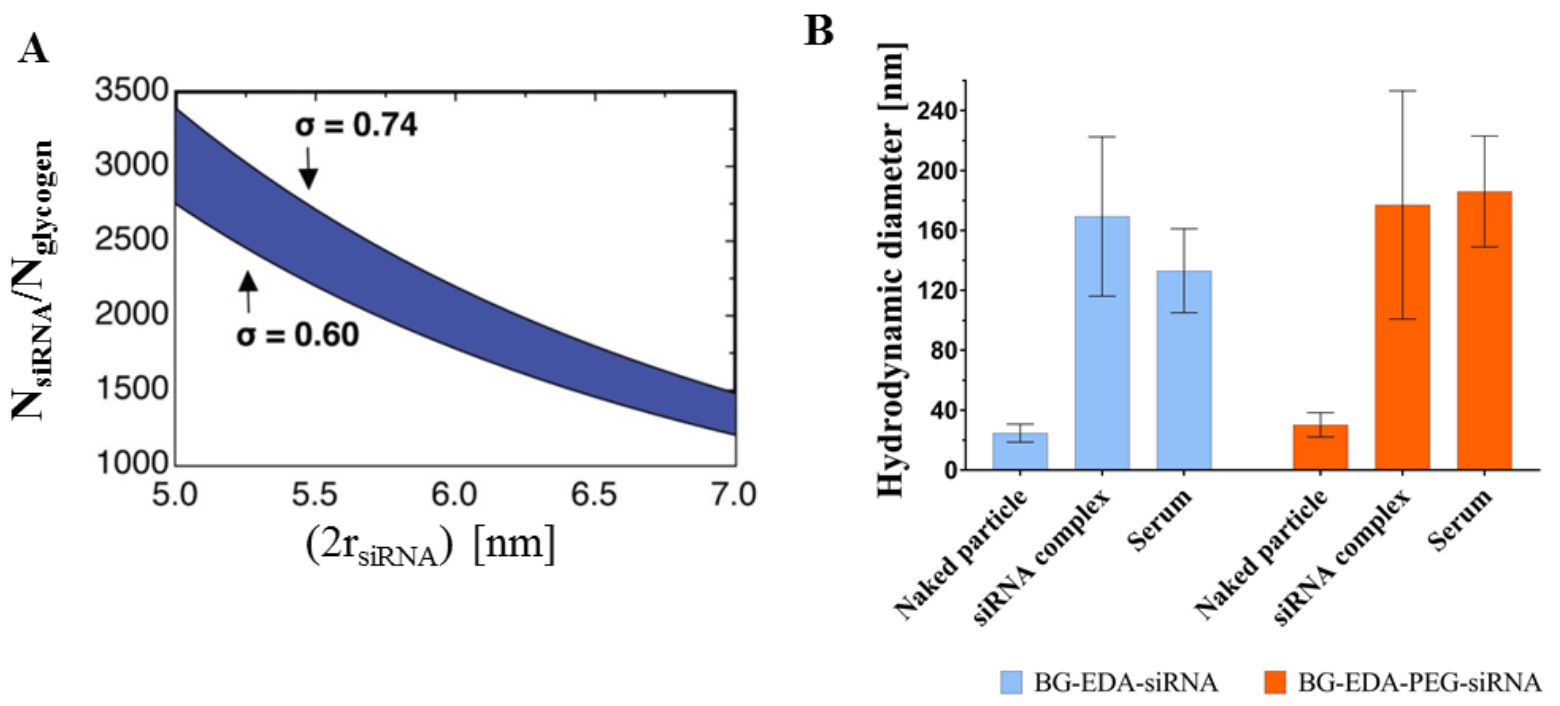
Figure 2. Structural and functional properties of glycogen-siRNA constructs. A) Approximate number of siRNA molecules per glycogen-siRNA construct that varies with the changing diameter of the siRNA molecules and also with the packing density within the glycogen-siRNA construct. B) Nanoparticle tracking and STORM size analysis of BG-EDA and BG-EDA-PEG and their siRNA constructs.

Table 2 summaries size distributions determined by DLS and nanoparticle tracking analysis (NTA) shown in Figure 2B. AFM imaging and analysis (Figure S7) shows that the dehydrated BG-EDA and BG-EDA-PEG-siRNA constructs maintain their integrity, with partial aggregation induced by the drying process.

Table 2. Size distributions of BG-EDA-siRNA and BG-EDA-PEG-siRNA constructs determined by DLS and NTA analysis

\begin{tabular}{cccc}
\hline \multirow{2}{*}{ Construct } & DLS & NTA \\
\cline { 2 - 4 } & $D_{\mathrm{h}}(\mathrm{nm})$ & PDI & $D_{\mathrm{h}}(\mathrm{nm})$ \\
\hline BG-EDA & $115 \pm 65$ & 0.43 & $160 \pm 53$ \\
BG-EDA-PEG & $125 \pm 78$ & 0.51 & $170 \pm 66$ \\
\hline
\end{tabular}

\subsection{In vitro and In Vivo Behavior of BG-EDA-siRNA- and BG-EDA-PEG-siRNA-Based Constructs}

To optimize the in vitro and in vivo properties of BG-EDA-siRNA and BG-EDA-PEGsiRNA constructs, their stability in serum must be assessed. The rapid disassembly of the construct by competitive serum proteins and hydrolytic enzymes may compromise its efficacy in delivering active siRNA.

The stability and integrity of constructs in the bovine serum proteins was verified by using STORM image analysis. We first prepared BG-EDA and BG-EDA-PEG-siRNA constructs tagged with the photoswitchable Alexa Fluor-647 dye. Next, we incubated the constructs in DMEM medium supplemented with $10 \%$ FBS for $5 \mathrm{~h}$. Imaging and analysis of the constructs adsorbed on glass coverslip show monodisperse particle populations for both samples (Figure S8), with particle sizes centered at around $133 \pm 28 \mathrm{~nm}$ and $186 \pm 37 \mathrm{~nm}$ for BGEDA-siRNA and BG-EDA-PEG-siRNA constructs, respectively (Figure 2B). These results 
are in agreement with DLS and NTA measurements performed in PBS and indicate that neither aggregation nor disassembly of constructs take place upon incubation with serum proteins.

To further verify if presence of serum proteins cause siRNA molecules to leak from the glycogen-siRNA constructs, a polyacrylamide gel electrophoresis study was performed. The polyacrylamide gel electrophoresis data (Figure S9), obtained after incubation of glycogensiRNA constructs with $20 \%$ FBS solution for $2 \mathrm{~h}$ at $37^{\circ} \mathrm{C}$, show that siRNA mainly remained immobilized in the construct (Figure S9 lane 4) and a negligible displacement of siRNA was induced (Figure S9 comparison between lane 1 and lane 4) by negatively charged serum proteins. To rule out the possibility that siRNA embedded in the construct is degraded by serum RNAse, the constructs were incubated with 25\% heparin. Heparin is a highly negatively charged oligosaccharide, which at high concentration is known to compete and displace siRNA in polyplexes [34]. This therefore allows determination of whether the siRNA in the construct remained intact after incubation with FBS. When incubated with heparin (Figure S9 lane 5) and FBS/heparin (Figure S9 lane 6), the constructs released intact double stranded siRNA.

Unmodified glycogen is a biodegradable polymer. Hence, circulating glucosidases in the serum may alter the integrity of BG-EDA-siRNA and BG-EDA-PEG-siRNA constructs. The degradability of BG-EDA and BG-EDA-PEG was investigated using an $\alpha$-amylase digestion assay. As previously reported [45], BG particles are degraded up to $50 \%$ by $\alpha$-amylase from Asperigillus oryzae upon incubation in PBS buffer (pH 7.2) at $25{ }^{\circ} \mathrm{C}$ for $3 \mathrm{~h}$. After modification, BG-EDA and BG-EDA-PEG were found to be resistant to $\alpha$-amylase digestion undergoing only $14 \%$ and $11 \%$ degradation, respectively (Figure S10). This decrease in degradability may be caused by a change in substrate recognition by $\alpha$-amylase due to the chemical modification of glucose, which may reduce enzyme accessibility.

Taken together, these results show that the engineered glycogen-siRNA constructs are likely capable of overcoming undesirable interactions with serum proteins. Although some molecules of siRNA may be released as a result of the constructs rearrangement in serum, the constructs maintain the siRNA payload. It is worth noting that previous estimates reported that less than 2000 siRNA molecules per cell are capable of causing maximal knockdown [13]; hence, one or two constructs must be effectively internalized into a cancer cell to deliver a therapeutic dose.

Glycogen-siRNA constructs were engineered with a spatial arrangement of hydrophilic polysaccharide brush-like residues and PEG arms to prolong in vivo blood circulation. It is well established that after systemic administration, the majority of circulating nanoparticles are non-specifically captured by macrophages and specialized endothelial cells residing in the liver and spleen [46,47]. In particular, resident macrophages of the liver, called Kupffer cells, are the predominant cells responsible for phagocytosis of many classes of circulating nanoparticles [48]. To investigate the in vivo behavior of our systems, BG-EDA- and BGEDA-PEG-siRNA constructs were intravenously injected into mice at a concentration of 1 
$\mathrm{mg} \mathrm{mL}^{-1}$ (the equivalent of $5 \mathrm{mg} / \mathrm{kg}$ of polymer/mouse by considering $20 \mathrm{~g}$ of mouse weight). Following the tail vein injection, the mice were imaged at different time intervals from 5 min to $24 \mathrm{~h}$ (Figure 3A). A non-invasive near-infrared fluorescence (NIRF) imaging approach was used to monitor the in vivo biodistribution of both constructs labeled with a CY7.5 NIRF dye. To monitor the fate of the constructs, we labeled the glycogen nanoparticles component as the labeling of a degradable molecule such as siRNA does not provide useful information. At $24 \mathrm{~h}$ post-injection, all mice were euthanized and major organs extracted for ex vivo NIRF imaging to determine the tissue distribution of labeled glycogen (Figure 3B).

Soon after injection, a visceral fluorescence signal for BG-EDA-siRNA construct (Figure 3C, red line) was observed, which increased in the first $2 \mathrm{~h}$, followed by a progressive decrease in signal. BG-EDA-PEG-siRNA construct showed evidence of visceral accumulation only after $2 \mathrm{~h}$ circulation (Figure 3C, black line), followed by a progressive increase with a maximum observed after $10 \mathrm{~h}$. These results may suggest a prolonged circulation in the blood for BG-EDA-PEG-siRNA constructs ultimately being sequestered by the visceral organs. After $10 \mathrm{~h}$ of circulation, the visceral fluorescence signals decreased, indicating the excretion of the constructs. As shown in Figure 3B, both constructs have high hepatic uptake and poor accumulation in the spleen. 

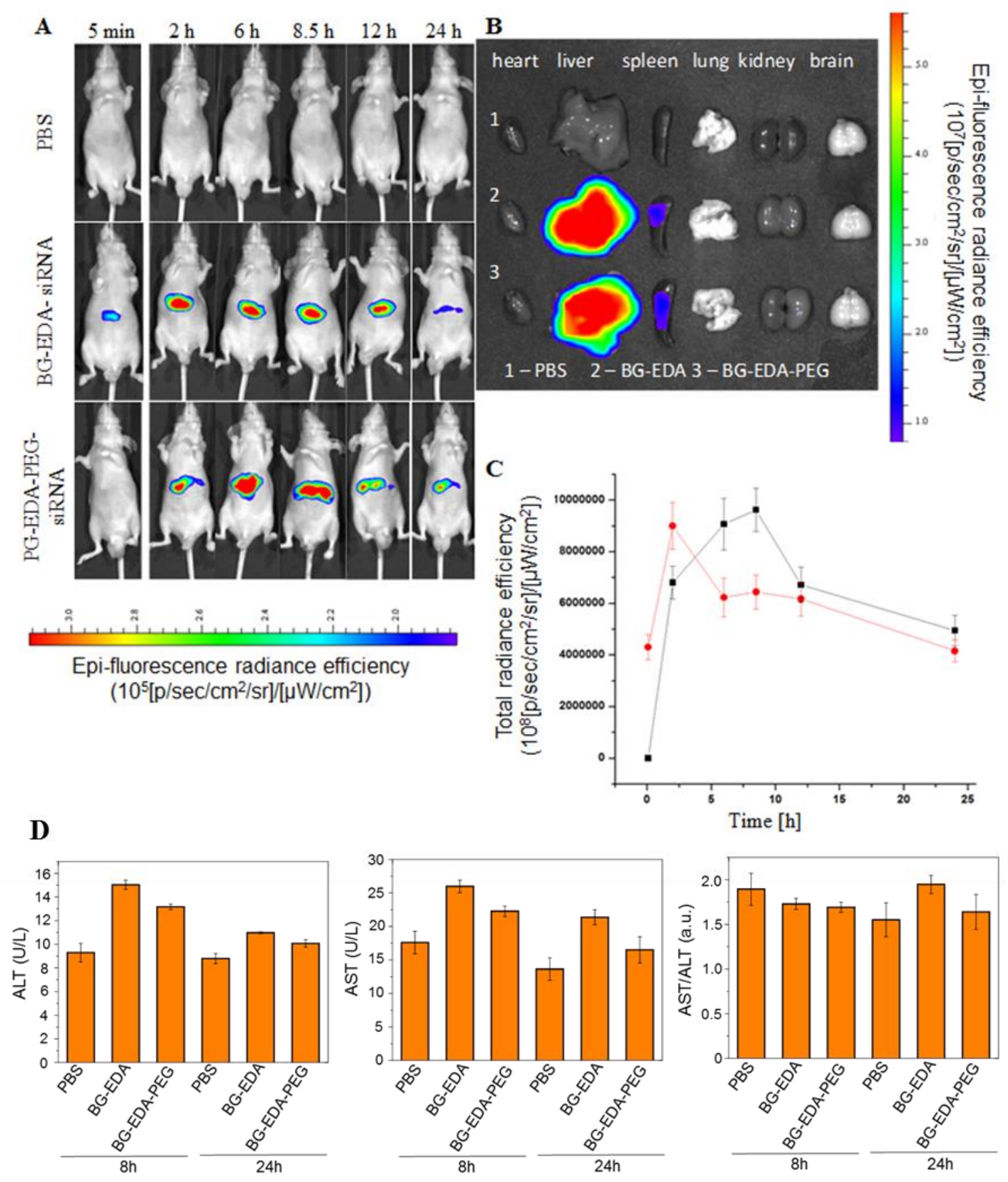

Figure 3. In vivo behavior of glycogen-siRNA constructs. A) Fluorescence images of the in vivo biodistribution of Cy7.5-labelled BG-EDA-siRNA and BG-EDA-PEG-siRNA constructs in male Balb/C mice at $24 \mathrm{~h}$ after intravenous injection of $100 \mu \mathrm{L}\left(1 \mathrm{mg} \mathrm{mL}{ }^{-1}\right)$. B) Fluorescence images of individual organs harvested $24 \mathrm{~h}$ after injection of Cy7.5-labelled BG-EDA-siRNA and BG-EDA-PEG-siRNA constructs. C) Glycogen-siRNA constructs liver accumulation profile over time (red: BG-EDA-siRNA; black: BG-EDA-PEG-siRNA). D) Liver toxicity evaluation by measuring levels of liver-related enzymes: alanine aminotransferase (ALT) and aminotransferase (AST) in serum after $8 \mathrm{~h}$ and $24 \mathrm{~h}$. 
This is likely due to active phagocytosis by macrophages in the liver. However, we cannot rule out a possible specific interaction with hepatocytes. The presence of fluorescence in the feces (Figure S11) suggests hepatobiliary excretion of constructs [48]. Interestingly, no evidence of accumulation in the lungs, kidney, heart, or brain was observed. The lack of significant lung-associated activity suggests that because of the small size, the constructs are devoid of non-specific interactions with lung capillaries, which typically cause accumulation in lungs. Indeed for the majority of other polyplexes reported in the literature for gene therapy, in vivo studies show that more than $50 \%$ of the total fluorescence was localized in the kidney [49,50]. This is because unstable polyplexes are rapidly disassembled in the kidney and therefore have higher kidney accumulation. In addition, as the polyplexes are formed through electrostatic interactions, competition with electrolytes, polyanions, or polycations present within the bloodstream may destabilize the nanoparticles, which are eliminated by renal clearance $[48,49]$. The lack of fluorescence signal in the kidneys in our study indicates that the engineered constructs are too large for kidney clearance into the urine. Importantly, there is no evidence of disassembly of glycogen-siRNA constructs in the kidney. In fact, we were not able to measure fluorescence in the urine and only a very weak signal was observed in the blood of mice injected with BG-EDA-PEG-siRNA constructs. However, the fluorescence signal in the body fluids may be below the detection limit of the instrument.

After injection of the constructs, all mice survived the $24 \mathrm{~h}$ study with no noticeable deterioration in health. All mice were active and showed no signs of lethargy or ill health after being dosed with up to $0.1 \mathrm{mg}$ of glycogen per mouse. To assess the liver toxicity after treatment with BG-EDA-siRNA and BG-EDA-PEG-siRNA constructs, we performed an assay to measure the levels of ALT and aminotransferase AST in serum after $8 \mathrm{~h}$ and $24 \mathrm{~h}$. As shown in Figure 3D, ALT and AST exhibited a slight enhancement in the treated mice in comparison to the control group, but were still in the normal range, while the AST/ALT value did not show obvious reduction after treatment with BG-EDA-siRNA or BG-EDA-PEGsiRNA constructs. H\&E staining of the liver sections (Figure S12) also did not show obvious changes that might indicate inflammation, necrosis,s or apoptosis of the liver, regardless of whether the mice were treated with BG-EDA-siRNA or BG-EDA- PEG-siRNA constructs. These studies indicate the minimal damage to the liver and good biocompatibility of both BG-EDA-siRNA and BG-EDA- PEG-siRNA constructs.

Overall, the in vivo studies revealed that glycogen-siRNA constructs are able to circulate in the blood for up to $8 \mathrm{~h}$ without toxicity and eventually accumulate in the liver. It is worth noting that to date, the biodistribution of glycogen nanocarriers in vivo has not been investigated, to the best of our knowledge. The present study has revealed the preferential accumulation of functional glycogen nanoparticles in the liver of healthy mice. 


\subsection{Intracellular Trafficking and Endosomal Escape of BG-EDA-siRNA and BG-EDA- PEG-siRNA Constructs}

To demonstrate the ability of the optimized construct (BG-EDA-siRNA w/w 4:1; BG-EDAPEG-siRNA w/w 6:1) to silence a set of different proteins by siRNA delivery, we used HEK293T cells expressing firefly luciferase and PC3 human prostate cancer cells overexpressing survivin as model cells. We first studied the cytotoxicity of the modified glycogen particles. The in vitro cytotoxic effect of the polymeric building blocks, RG-EDA, OG-EDA, BGEDA, BG-EDA-PEG, was evaluated by an MTT assay upon $24 \mathrm{~h}$ incubation (Figure S13) with the selected model cell lines. Cell viability was unaffected by incubation with BG-EDA and BG-EDA-PEG in the concentration range studied (up to $0.5 \mathrm{mg} \mathrm{mL}^{-1}$ ), whereas we observed cytotoxicity (20\%) for RG-EDA and OG-EDA nanoparticles at $0.12 \mathrm{mg} \mathrm{mL}^{-1}$.

A number of studies have shown that the molecular weight of linear and branched polycations, as well as the net surface charge and charge density, contribute to the onset of cell death by rupture of the cell membrane [51]. Higher cytotoxicity of these synthetic polymers is observed as the molecular weight of the polymer increases, likely due to the high flexibility of the charged polymeric chains that can potentially rupture the cell membrane [52], and the localization of the non-degradable polymers in the nuclei, where they may interfere adversely with the host genes [53]. Unlike the gold standard of polycationic gene delivery vectors - PEI, which is toxic at concentrations higher than $0.03 \mathrm{mg} \mathrm{mL}^{-1}$ (Figure S14), glycogen-siRNA constructs and in particular BG-EDA and BG-EDA-PEG show a desirable toxicity profile even at high concentrations $\left(0.5 \mathrm{mg} \mathrm{mL}^{-1}\right)$. This can be explained by the dendritic but compact architecture of cationic glycogen being less prone to entanglement of its "tree-like" chains into the hydrophobic lipid membranes of cells.

We evaluated the association of fluorescently labeled (AF-488) BG-EDA-siRNA and BGEDA-PEG-siRNA constructs with PC3 and 293T cells as a function of time using flow cytometry (Figure S15). In PC3 cells (Figure S15A), we observed rapid association of constructs in the first $8 \mathrm{~h}$ of incubation. The association takes into account both membrane binding and intracellular uptake processes. A significantly higher and faster association was measured for BG-EDA-siRNA constructs compared to BG-EDA-PEG-siRNA constructs. We speculate that both the larger size and stealth properties of the PEG chains may slow down the uptake process of the BG-EDA-PEG-siRNA constructs. In 293T cells (Figure S15B), similar association profiles were observed for both systems; however, a plateau was not observed, indicating a progressive association and uptake of constructs after $24 \mathrm{~h}$ of incubation. This highlights the importance of verifying the efficacy of gene nanocarriers in different cell lines.

To gain insight into the cell internalization and trafficking of glycogen-siRNA constructs, cells were analyzed using confocal microscopy. The major internalization mechanism of lipoplexes and polyplexes is micropinocytosis [54]. Upon uptake, the nanocarriers are transported through the early endosomes, late endosome and lysosomes, depending on the physicochemical properties [55]. The various stages of trafficking can be evaluated by 
analyzing the co-localization with specific bio-markers, such as EEA1 and Rab7, which are used to identify early endosomes and late endosomes/lysosomes, respectively. Rab7 is used to monitor trafficking between late endosomes and lysosomes, where Rab7 positive vesicles can be considered terminal vesicles in the endo-lysosomal pathway. The internalization and trafficking of glycogen-siRNA constructs into PC3 and 293T cells were studied using antibodies for these specific markers. Analyses of confocal microscopy images taken after $4 \mathrm{~h}$ incubation of glycogen-siRNA constructs with PC3 and 293T cells (Figure 4) show effective internalization of BG-EDA-siRNA and BG-EDA-PEG-siRNA construct (green dots). Confocal microscopy images (Figure 4) acquired from the two different cell lines show limited co-localization (yellow dots) of BG-EDA-siRNA and BG-EDA-PEG-siRNA constructs within both early and late endosomes/lysosomes (red dots). 


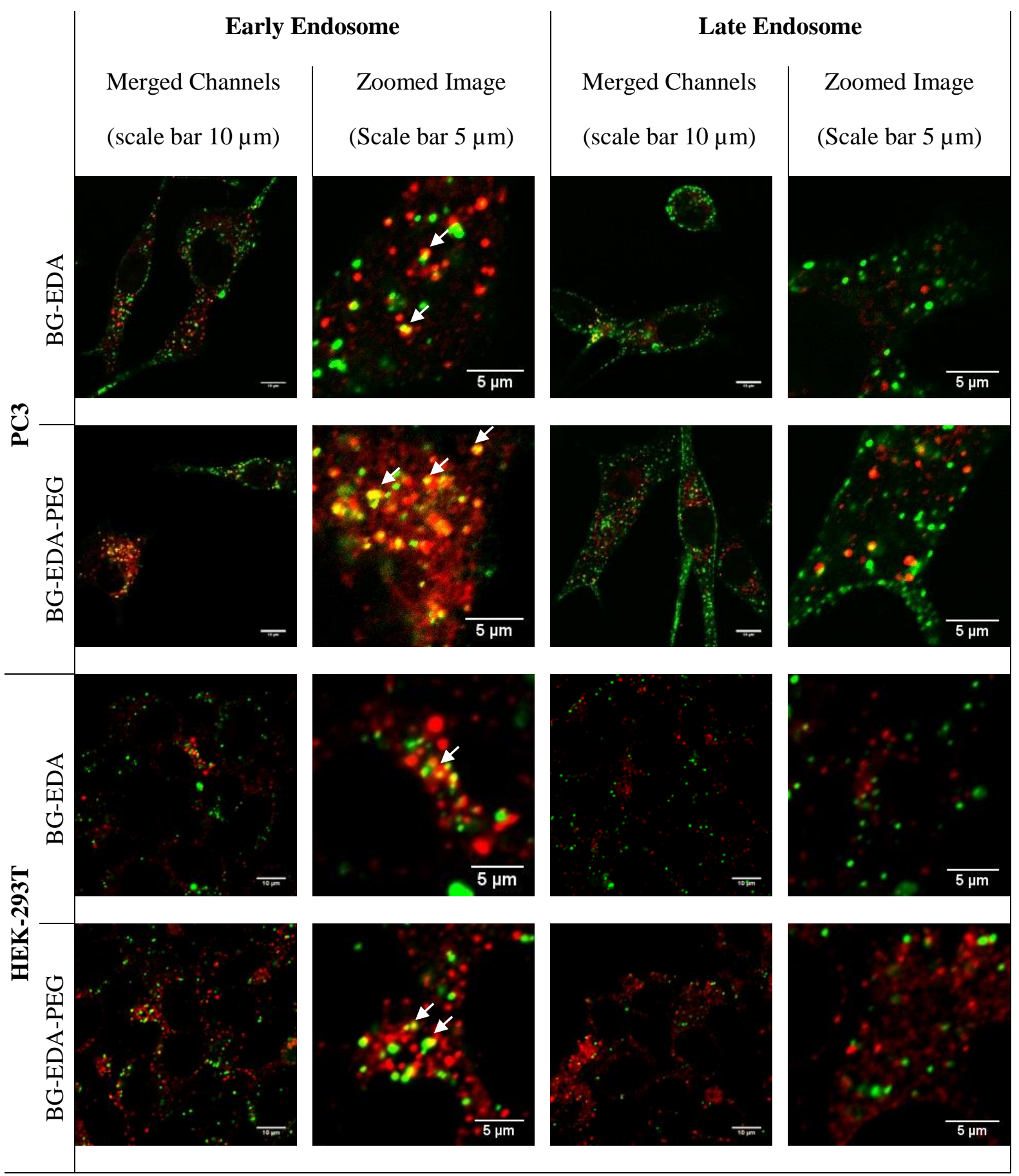

Figure 4. Intracellular trafficking of glycogen-siRNA constructs. Co-localization studies of constructs in PC3 and HEK-293T cells with confocal laser scanning microscopy. Images were taken $4 \mathrm{~h}$ after transfection with AF-488 labeled BG-EDA-siRNA and BG-EDA-PEGsiRNA constructs (green). Endosomes (red) were stained with mouse anti-EEA1 monoclonal antibody (early endosome) or rabbit anti-Rab7 monoclonal antibody (late endosome) followed by a secondary Alexa Fluor-647 conjugated antibody. 
This is a clear indication of endosomal escape and cytosolic localization of constructs after 4 $\mathrm{h}$ of incubation. Pearson's correlation function (PCC) (Table S2) was used to quantify colocalization of the constructs. BG-EDA-siRNA and BG-EDA-PEG-siRNA constructs display a PCC value of approximately 0.2 in both tested cell lines after staining with anti-EEA1 antibody, indicating a low degree of co-localization with early endosomes. The PCC value for late endosome co-localization is 0.07 , suggesting no co-localization of both constructs with this compartment.

The escape of siRNA from the endosome into the cytosol is usually considered as a ratelimiting step in nucleic acid delivery approaches. To address this hurdle, we developed a few strategies. The proton sponge effect, an endosomal escape mechanism, is deemed to depend on the buffering properties of BG-EDA possessing a $\mathrm{pKa}$ in the range of the endosomal $\mathrm{pH}$ (step 2 in Figure 5). After endocytosis, the endosomal interior is acidified because of the activity of the ATP-dependent proton pump. This leads to a change in $\mathrm{pH}$ inside the vesicle from 7.2 to 6.3 in the early endosome and to 5.5 in the late endosome [54]. We carefully selected EDA to modify the glycogen because of its potential to trigger the proton sponge effect via the secondary amine and to complex siRNA via the primary amine groups. We measured the pKa values of BG-EDA by potentiometric titration and found that BG-EDA has two pKa values (Figure S16) i.e., pKa 6.4 and pKa 2 about 10. The partial degradability of BG-EDA and BG-EDA-PEG is the second strategy we put in place to promote the endolysosomal escape of the construct. It is known that native glycogen is degraded inside lysosomes by $\alpha$-glucosidase (GAA) (step 4 in Figure 5) [56].

We hypothesize that a mild degradation of glycogen-siRNA into oligosaccharides and glucose could burst the lysosomal compartments by inducing an osmotic pressure gradient (step 7 in Figure 5). The combination of the proton sponge effect and osmotic pressure mechanisms is possibly conferring an endo-lysosomotropic property to the constructs. Indeed, this prediction is supported by the confocal imaging data and further by the genesilencing efficacy of the construct. 


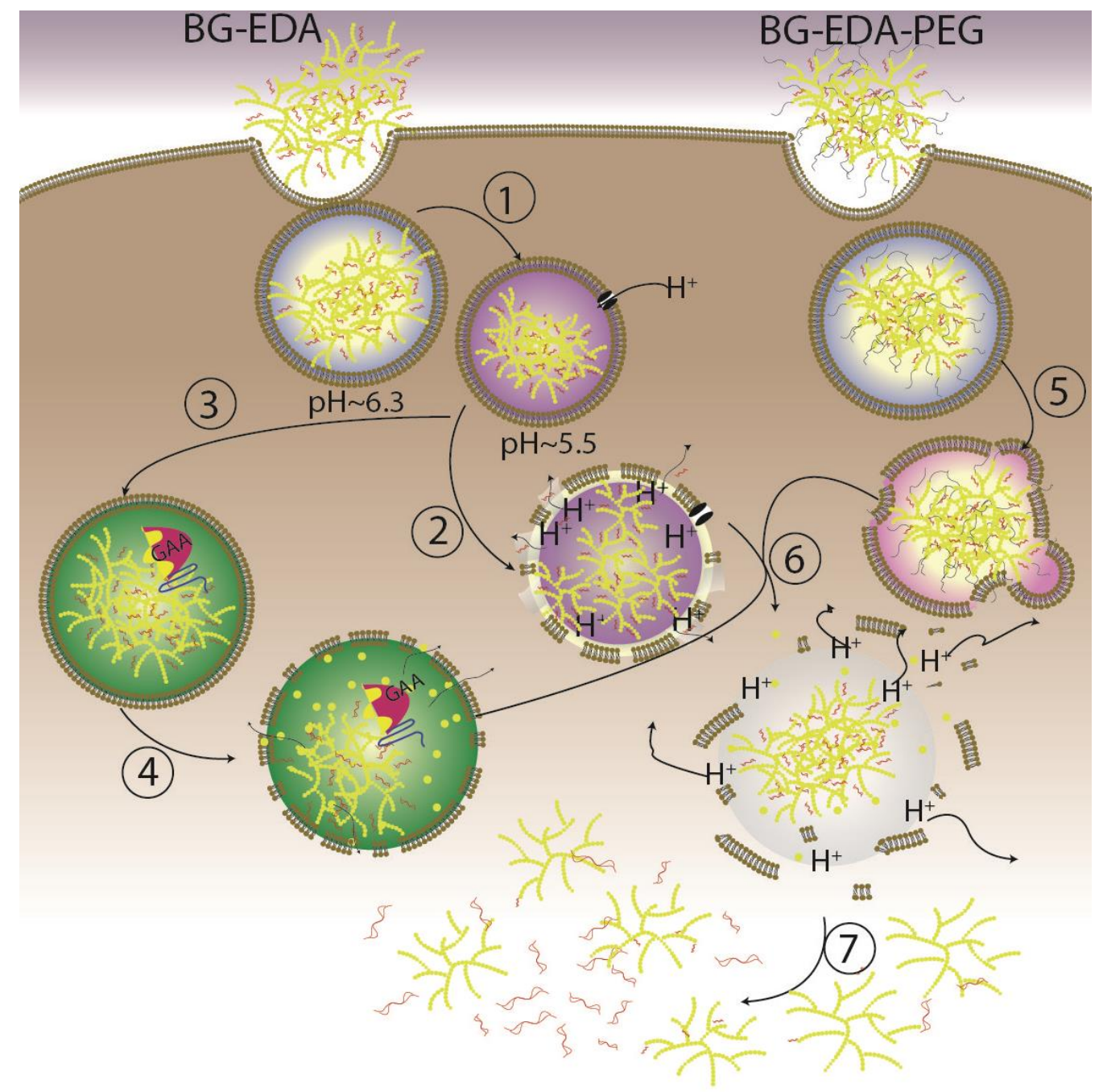

Figure 5. Schematic representation of the proposed endosomal escape mechanism for glyocgen-siRNA constructs. After endocytosis, the construct enters the late endosome (step 1), which either activates the proton sponge effect (step 2) or proceeds to the lysosome (step 3) and escapes into the cytosol owing to the lysomotropic effect of lysosomal alphaglucosidase (GAA) on glycogen (step 4). The PEG-modified-construct may induce membrane rupture by fusion with membrane lipids (step 5). Each strategy alone or in combination leads to the final endosome/lysosome escape of the constructs (step 6) and disassembly inside the cytosol (step 7).

\subsection{In vitro Gene Silencing of BG-EDA-siRNA and BG-EDA-PEG-siRNA Constructs}

The knockdown efficacy of the constructs was evaluated in monolayer cultured 293T and PC3 cell lines by targeting luciferase (293T cells) and survivin (PC3 cells) proteins. The $293 \mathrm{~T}$ cell line used in our studies is modified with a luciferase reporter vector that expresses firefly luciferase, which catalyzes the oxidation of beetle luciferine into a luminescent product. The luminescence intensity is strongly correlated to the amount of luciferase inside the cell and allows for easy monitoring of protein expression. Survivin belongs to the family 
of inhibitors of apoptosis proteins, and its expression is highly upregulated in most carcinomas [57].

Cells were incubated for $6 \mathrm{~h}$ with siRNA packaged into BG-EDA-siRNA (w/w 4:1) and BGEDA-PEG-siRNA (w/w 6:1) constructs and analyzed at different time points after transfection. Knockdown efficacy was evaluated using protein-specific immunostaining by flow cytometry and Western immunoblotting in PC3 cells, whereas luciferase downregulation was determined with a luminescence assay. The efficacy of BG-EDA and BG-EDA-PEG in siRNA transfection was compared to the commercially available in vitro cationic lipid-mediated transfection agent Lipofectamine RNAiMax. It is worth noting that Lipofectamine RNAiMax revealed significant toxicity on PC3 cells after $24 \mathrm{~h}$ incubation (Figure S17). Data in Figure 6A and 6B show the downregulation of protein expression after treatment with protein specific and scrambled siRNA-loaded BG-EDA and BG-EDA-PEG constructs up to $96 \mathrm{~h}$. We observed a time-dependent silencing effect of the proteins. At shorter incubation time, the same specific silencing at levels of $30 \%$ and $40 \%$ was detected for BG-EDA-siRNA and BG-EDA-PEG-siRNA constructs, respectively. At a longer incubation time (i.e. $96 \mathrm{~h}$ ), higher downregulation of protein expression was obtained, with up to $50 \%$ and $70 \%$ for BG-EDA- and BG-EDA-PEG-siRNA constructs, respectively. No significant downregulation was observed in cells treated with glycogen loaded with scrambled siRNA (negative control), indicating that the observed knockdown was specific. In addition, the negative control suggests that the glycogen-based transfection itself does not trigger off-target effects on the analyzed proteins. 

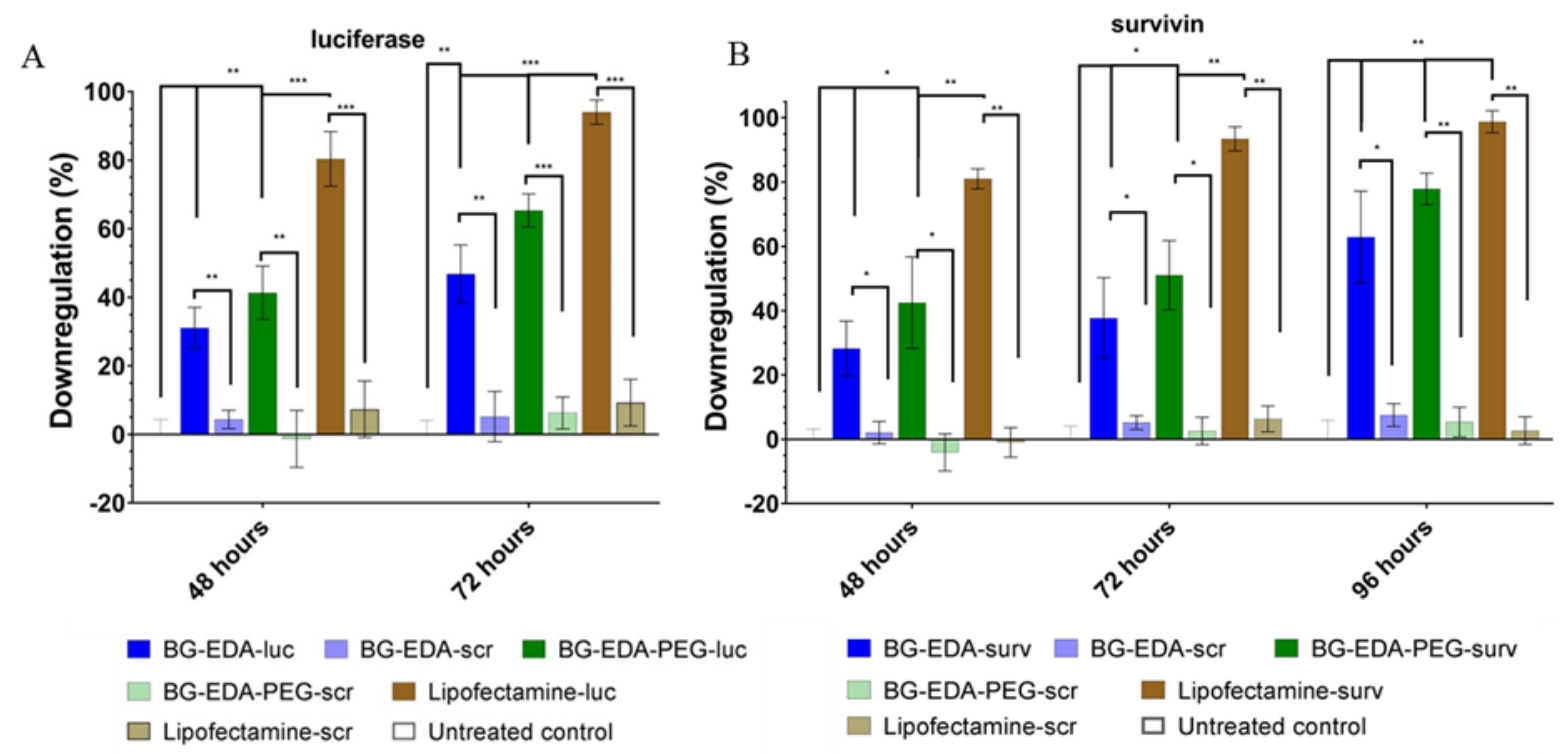

Figure 6. A) Luciferase (luc) gene knockdown in 293T-Luc cells 48 and $72 \mathrm{~h}$ after transfection. B) Survivin (surv) gene knockdown in PC3 cells 48 and $72 \mathrm{~h}$ after transfection. Error bars represent standard deviation. Statistical significance was determined by a twotailed $t$-test and is reported as $* p<0.05,{ }^{*} p<0.01$ and $* * * p<0.001$.

Western immunoblotting performed $72 \mathrm{~h}$ after transfection (Figure S18A) confirmed the survivin knockdown data obtained using flow cytometry. We also performed viability assays with Alamar Blue at each time point to evaluate the effect of transfection and survivin knockdown on cell proliferation and metabolic activity (Figure S18B). We did not observe any influence on cell viability after transfection with glycogen-siRNA constructs. It is known that a reduction in survivin level in cancer cells does not contribute to immediate cell death or reduced metabolic activity under standard culturing conditions due to overexpression of other proteins with similar functions [58,59]. It is worth noting that although BG-EDA-PEGsiRNA constructs have a lower cellular uptake in tested cell lines, they show a better silencing efficacy than BD-EDA-siRNA construct. This is likely due to the protective effect of BG-EDA-PEG exerted on siRNA molecules against hydrolytic enzymes and competitive serum proteins.

\subsection{Gene Silencing in 3D Tumor Spheroid Models}

As discussed earlier, a major barrier to reducing the in vivo therapeutic efficacy of siRNA delivery is the restriction of interstitial transport, which obstructs the diffusion of nanoparticles into the solid tumor tissue. To determine whether the engineered glycogensiRNA constructs are amenable to solid tumor penetration and in vivo gene delivery, we studied the ability of BG-EDA-siRNA and BG-EDA-PEG-siRNA constructs to effectively penetrate the ECM and access the cells in MCTS. The 293T cells were seeded on a culture plate covered with ultra-low attachment coating and left to grow for 5 days. PC3 cells were 
seeded similarly and left to grow for at least 8 days. The 293T and PC3 spheroids vary in morphology. Fully associated and compact tissue-like structures were obtained after 3 days of culture of 293T cells. The assembly of PC3 cells into spheroids required longer incubation time because of poorer formation of cell junctions. As a consequence, less dense and loosely adhered spheroids were obtained. Mature spheroids of around $500 \mu \mathrm{m}$ in size were used to assess whether glycogen-siRNA constructs were able to penetrate the spheroids and be internalized into the cells. Figure 7A presents the microtome cross-sections of a spheroid analyzed by confocal microscopy at increasing depths.

Figure 7A and Figure S19 show that BG-EDA-siRNA constructs effectively penetrate 293T spheroids and accumulate predominantly in the spheroid core. BG-EDA-PEG-siRNA constructs (Figure 7A and Figure S20) are also able to penetrate the tumor; however, large amounts of particles remain associated on the outer layer of the spheroids. The different diffusional and penetration behaviors can be correlated to the larger BG-EDA- and BG-EDAPEG-siRNA constructs formed. Accumulation of the constructs in the spheroid core can be ascribed to a higher cell density within the spheroid core when compared with the outer layer of the spheroid, effective particle internalization, and rapid diffusion of the constructs across the entire spheroid. Compared to 293T spheroids, PC3 spheroids exhibited a more even spread or uniform penetration of BG-EDA-siRNA constructs across the sections (Figure 7A). The differences in diffusional patterns between 293T and PC3 spheroids arise from these spheroid systems significantly varying in their morphology. As previously mentioned, PC3 cells form less compact spheroids, with a rather uniform cell density across the entire sphere, making glycogen constructs evenly diffused throughout the spheroid. In contrast, 293T cells create spheroids with a high cell density core, in comparison with their outer shell, that accumulate glycogen constructs within the core. Images taken at a higher resolution revealed the cellular localization of BG-EDA-siRNA and BG-EDA-PEG-siRNA constructs. This provides evidence that glycogen constructs not only penetrate the spheroids ECM, but also are effectively internalized into the tumor cells.

To verify the ability of BG-EDA-siRNA and BG-EDA-PEG-siRNA constructs to silence genes within spheroids, we evaluated luciferase downregulation in 293T spheroids as well as survivin in PC3 spheroids $72 \mathrm{~h}$ post-transfection in 2D cell cultures. Prior to each assay, spheroids were dissociated to single cells with Accutase. BG-EDA-siRNA and BG-EDAPEG-siRNA constructs (Figure 7B and C) both exhibited $40 \%$ and $60 \%$ knockdown for luciferase and survivin expression, respectively. The level of downregulation caused by glycogen-siRNA constructs in spheroids is comparable with the data obtained in 2D cultures (Figure 6A and B). 
A $\sim 60 \mu \mathrm{m}$ $\sim 250 \mu \mathrm{m}$ $\sim 480 \mu \mathrm{m}$ $\sim 250 \mu \mathrm{m}$
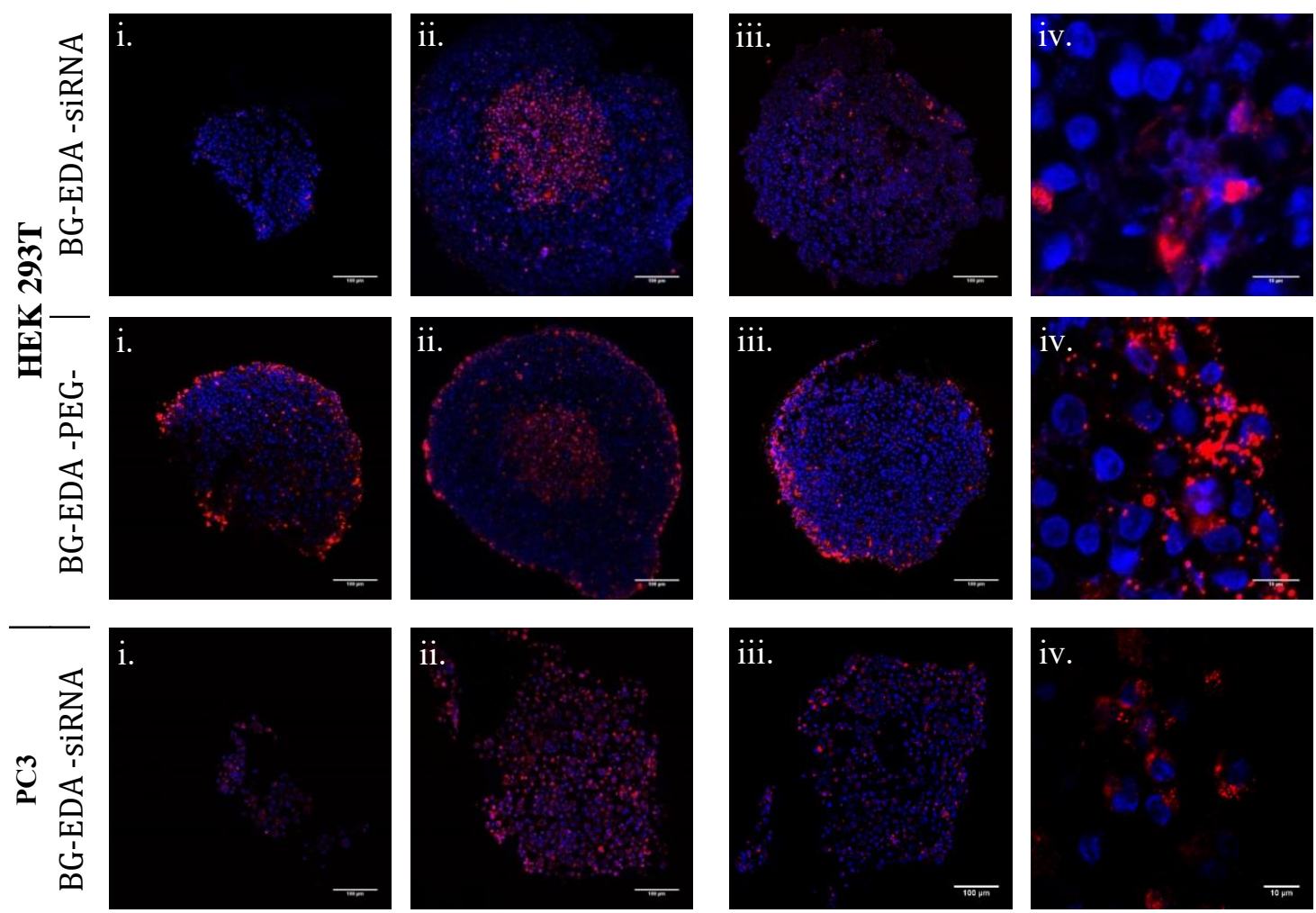

B

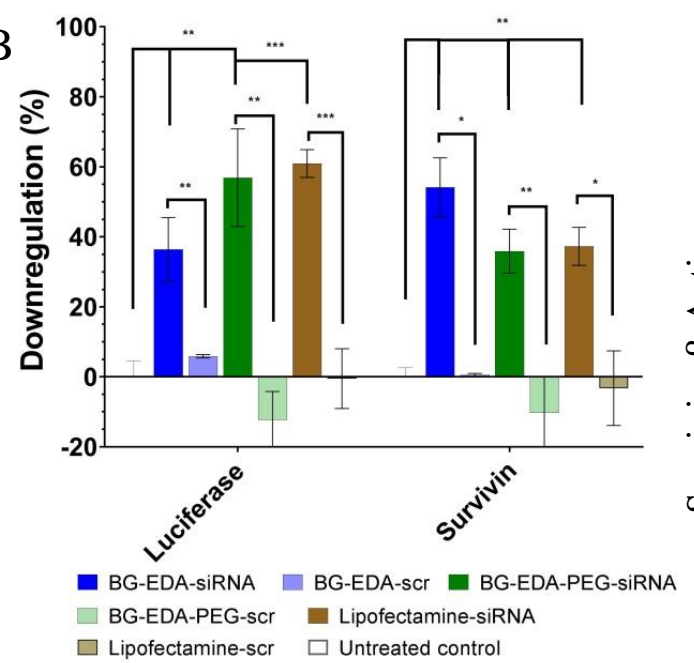

$\mathrm{C}$

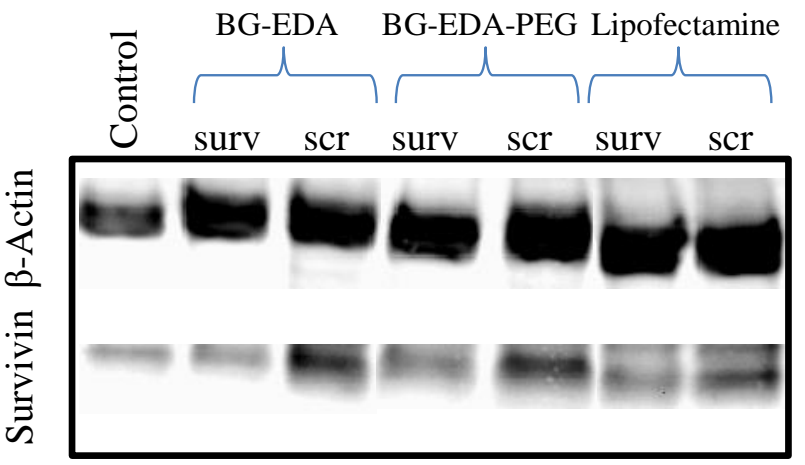

Figure 7. Penetration and gene silencing in spheroids. A) Confocal laser scanning microscopy images of sections of 293T and PC3 spheroids treated with BG-EDA-siRNA and BG-EDA-PEG-siRNA constructs for $24 \mathrm{~h}$ taken with a 20× (i-iii: scale bar $100 \mu \mathrm{m}$ ) and a 60× (iv: scale bar $10 \mu \mathrm{m}$ ) magnification objective at increasing spheroid depth. Blue, nucleus; Red, particles. B) Luciferase and survivin knockdown in 293T and PC3 spheroids $72 \mathrm{~h}$ posttransfection. Error bars represent standard deviations. Statistical significance was determined by a two-tailed $t$-test and is reported as $* p<0.05, * * p<0.01$ and $* * * p<0.001$. C) Representative Western Blot of survivin knockdown in PC3 spheroids. 
Enhanced knockdown efficacy of BG-EDA-PEG-siRNA constructs was also observed in spheroids. Lipofectamine was found to be less effective in specific gene silencing in 3D spheroids than in 2D cell culture. Reduced efficacy of Lipofectamine is due to the interaction of lipids with the spheroid outer shell [19]. As a result, in 3D spheroids, the gene silencing efficiency of the BG-EDA-siRNA and BG-EDA-PEG-siRNA constructs is comparable and even higher when compared with the silencing activity of Lipofectamine. Taken together, these results indicate that the glycogen-siRNA constructs can cross the barriers imposed by the 3D architecture of spheroids, readily diffuse inside the ECM network pores, and importantly, remain free inside the network without entrapment from nonspecific electrostatic interactions with extracellular proteins.

\section{Conclusions}

We engineered a new particle construct based on functionalized glycogen for the delivery of nucleic acids to tumor spheroids. Tumor spheroids were chosen as a cellular model system that features several in vivo characteristics of the tumor microenvironment, including high cell density, 3D architecture, cell-to-cell contacts, and production of the extracellular matrix network. The size and surface charge density of glycogen-siRNA constructs were finely controlled to minimize interactions with serum proteins and allow significant penetration into 3D multicellular spheroids of HEK-293T and PC3 cells. The glycogen-siRNA constructs, containing approximately 1200-3300 molecules of siRNA, were found to mediate the downregulation of luciferase and survivin in 293T and PC3 monolayer cell cultures, and 3D multicellular spheroids. The gene knockdown of the glycogen-siRNA constructs in 3D multicellular spheroids was comparable and even higher in comparison to the commercial transfection agent Lipofectamine RNAiMax. Confocal microscopy images indicate endosomal escape and cytosolic localization of glycogen-siRNA constructs after $4 \mathrm{~h}$ of incubation. An in vivo biodistribution study of the glycogen-siRNA constructs injected in mice revealed no cytoxicity and preferential accumulation in the liver, with no uptake into the spleen, lung, kidney, heart, and brain. BG-EDA-PEG-siRNA constructs exhibited a prolonged circulation lifetime of up to $8 \mathrm{~h}$. Taken together, our results highlight the importance of screening newly developed siRNA nanocarriers in 3D cell cultures for verifying their efficient siRNA release and emphasize the utility of glycogen as a versatile and promising platform for in vivo gene therapy.

Supporting Information. 1H-NMR spectra of glycogens and cationic glycogens, reaction scheme, SAXS data, cell viability assays, serum stability gel electrophoresis, association studies, Western immunoblotting, microtome sections, fluorescence imaging of bio-fluids.

\section{Author Contributions}

The manuscript was written through contributions of all authors. M.W., F.Cav., and F.Car. conceived the work. M.W. conducted the synthesis, structural, and bio-functional characterization of glycogen nanoparticles, the cell biology and flow cytometry work, siRNA 
transfection, immunostaining experiments, and data processing. Q.B. conducted the AFM and SAXS experiments. Y.L.W. and X.J.L. performed the in vivo experiments, J.A.B optimized the preparation of tumor spheroids, A.G. and C.C.J. conducted the gel electrophoresis experiments.. 37M.W, Q.B., F.Cav., and F.Car. drafted the manuscript. All authors discussed the results and commented on the manuscript, and have given approval to the final version of the manuscript.

\section{Acknowledgements}

This work was supported by the Australian Research Council (ARC) under a Future Fellowship (F. Cavalieri, FT140100873) and an Australian Laureate Fellowship (F. Caruso, FL120100030) schemes. This work was also supported by a University of Melbourne Establishment Grant (F. Cavalieri). This research was conducted by the ARC Centre of Excellence in Convergent Bio-Nano Science and Technology (project number CE140100036). This work was performed in part at the Materials characterization and Fabrication Platform (MCFP) at The University of Melbourne, the Victorian Node of the Australian National Fabrication Facility (ANFF), and the SAXS/WAXS beamline of the Australian Synchrotron, Victoria, Australia (11587). We thank Laura Leone for assistance with microtome sectioning.

\section{Data Availability}

The raw/processed data required to reproduce these findings cannot be shared at this time due to legal or technical reasons. 


\section{References}

1. A. Wittrup, J. Lieberman, Knocking Down Disease: a Progress Report on siRNA Therapeutics, Nat. Rev. Genet. 16 (2015) 543-552.

2. C. Lorenzer, D. Mehrdad, A.M. Winkler, V. Baumann, J. Winkler, Going Beyond the Liver: Progress and Challenges of Targeted Delivery of siRNA Therapeutics, J. Control. Release 203 (2015) 1-115.

3. L.M. Kranz, M. Diken, H. Haas, S. Kreiter, C. Loquai, K.C. Reuter, M. Meng, D. Fritz, F. Vascotto, H. Hefesha, C. Grunwitz, M. Vormeher, Y. Hussemann, A. Selmi, A.N. Kuhn, J. Buck, E. Derhovanessian, R. Rae, S. Attig, J. Diekmann, R.A. Jabulowsky, S. Heesch, J. Hassel, P. Langguth, S. Grabbe, C. Huber, O. Tureci, U. Sahin, Systemic siRNA Delivery to Dendritic Cells Exploits Defence for Cancer Immunotherapy, Nature 534 (2016) 396-401.

4. J. Conde, C.E. Arnold, F. Tian, N. Artzi, RNAi Nanomaterials Targeting Immune Cells as an Anti-Tumor Therapy: the Missing Link in Cancer Treatment?, Mater. Today 19 (2016) 2943.

5. S. Kamerkar, V.S. LeBleu, H. Sugimoto, S. Yang, C.F. Ruivo, S.A. Melo, J.J. Lee, R. Kalluri, Exosomes Facilitate Therapeutic Targeting of Oncogenic KRAS in Pancreatic Cancer, Nature 546 (2017) 498-503.

6. J. Conde, A. Ambrosone, Y. Hernandez, F. Tian, M. McCully, C.C. Berry, P.V. Baptista, C. Tortiglione. J.M. de la Fuente, 15 Years on siRNA Delivery: Beyond the State-of-the-Art on Inorganic Nanoparticles for RNAi Therapeutics, Nano Today 10 (2015) 421-450.

7. K. Garber, Worth the RISC?, Nat. Biotechnol. 35 (2017) 198-202.

8. H.J. Kim, A. Kim, K. Kataoka, Recent Progress in Development of siRNA Delivery Vehicles for Cancer Therapy, Adv. Drug Delivery Rev. 104 (2016) 61-77.

9. S.Y.C Choi, D. Lin, P.W. Gout, C.C. Collins, Y. Xu, Y. Wang, Lessons from PatientDerived Xenografts for Better In Vivo Modeling of Human Cancer, Adv. Drug Deliv. Rev. 79-80 (2014) 222-237

10. P. Zhang, K. An, X. Duan, H. Xu, F. Li, F. Xu. Recent Advances in siRNA Delivery for Cancer Therapy Using Smart Nanocarriers, Drug Discovery Today (2018), https://doi.org/10.1016/j.drudis.2018.01.042

11. C. Godfrey, L.R. Desviat, B. Smedsrod, F. Pietri-Rouxel, M.A. Denti, P. Disterer, S. Lorain, G. Nogales-Gadea, V. Sardone, R. Anwar, S.E.L. Andaloussi, T. Letho, B. Khoo, C. Brolin, W.M.C. van Roon-Mom, A. Goyenvalle, A. Aartsma-Rus, V. Arechavala-Gomeza, Delivery Is Key: Lessons Learnt from Developing Splice-Switching Antisense Therapies, EMBO Mol. Med. 9 (2017) 545-557 
12. J.E. Zuckerman, M.E. Davis, Clinical Experiences with Systematically Administrated siRNA-Based Therapeutics in Cancer, Nat. Rev. Drug Discovery 14 (2015) 843-856

13. A. Wittrup, A. Ai, X. Liu, P. Hamar, R. Trifonova, K. Charisse, M. Manoharan, T. Kirchhausen, J. Liberman, Visualaising Lipid-Formulated siRNA Release from Endosomes and Target Gene Knockdown, Nat. Nanotechnol. 33 (2015) 870-878.

14. S. Wilhelm, A.J. Tavares, Q. Dai, S. Ohta, J. Audet, H.F. Dvorak, W.C. Chan, Analysis of Nanoparticle Delivery to Tumors, Nat. Rev. Mater. 1 (2016) 1-12.

15. M. Zanoni, F. Piccinini, C. Arienti, A. Zamagni, S. Santi, R. Polico, A. Bevilacqua, A. Tesei, 3D Tumor Spheroid Models for In Vitro Therapeutic Screening: A Systematic Approach to Enhance Biological Relevance of Data Obtained, Sci. Rep. 6 (2016) 19103.

16. W. Shi, D. Weng, W. Niu, Nanoparticle Drug Delivery Systems and Three-Dimensional Cell Cultures in Cancer Treatments and Research, Cancer Transl. Med. 2 (2016) 154-161.

17. J. Friedrich, C. Seidel, R. Ebner, L.A. Kunz-Schughart, Spheroid-Based Drug Screen: Considerations and Practical Approach, Nat. Protoc. 4 (2009) 309-324.

18. M. Oishi, Y. Nagasaki, N. Nishiyama, K. Itaka, M. Takagi, A. Shimamoto, Y. Furuichi, K. Kataoka, Enhanced Growth Inhibition of Hepatic Multicellular Tumor Spheroids by Lactosylated Poly(ethylene glycol)-siRNA Conjugate Formulated in PEGylated Polyplexes, ChemMedChem 2 (2007) 1290-1297.

19. C. Carver, X. Ming, R.L. Juliano, Multicellular Tumor Spheroids as a Model for Assessing Delivery of Oligonucleotides in Three Dimensions, Mol. Ther. Nucleic Acids 3 (2014) e153.

20. C.L. Waite, C.M. Roth, PAMAM-RGD Conjugates Enhance siRNA Delivery through a Multicellular Spheroid Model of Malignant Glioma, Bioconjug. Chem. 20 (2009) 1908-1916.

21. T.T. Goodman, J. Chen, K. Matveev, S.H. Pun, Spatio-Temporal Modeling of Nanoparticle Delivery to Multicellular Tumor Spheroids, Biotechnol. Bioeng. 101 (2008) 388-399

22. X. Xu, C.R. Sabanayagam, D.A. Harrington, M.C. Farach-Carson, X. Jia, A HydrogelBased Tumor Model for the Evaluation of Nanoparticle-Based Cancer Therapies, Biomaterials 35 (2014) 3319-3330

23. Y. Jiang, H. Lu, A. Dag, G. Hart-Smith, M.H. Stenzel, Albumin-Polymer Conjugate Nanoparticles and Their Interactions with Prostate Cancer Cells in 2D and 3D Culture: Comparison Between PMMA and PCL, J. Mater. Chem. B 4 (2016) 2017-2027

24. K. Kostarelos, D. Emfietzoglou, A. Papakostas, W.H. Yang, A.M. Ballangrud, G. Sgouros, Engineering Lipid Vesicles of Enhanced Intratumoral Transport Capabilities: 
Correlating Liposome Characteristics with Penetration into Human Prostate Tumor Spheroids, J. Liposome Res. 15 (2005) 15-27

25. G.A. Morris, S. Ang, S.E. Hill, S. Lewis, B. Schafer, U. Nobbmann, S.E. Harding, Molar Mass and Solution Conformation of Branched alpha(1 - 4), alpha(1 - 6) Glucans. Part I: Glycogens in Water, Carbohydr. Polym. 71 (2008) 101-108.

26. W. Burchard, Structure Formation by Polysaccharides in Concentrated Solution, Biomacromolecules 2 (2001) 342-353.

27. J.L. Putaux, A. Buleon, R. Borsali, H. Chanzy, Ultrastructural Aspects of Phytoglycogen from Cryo-Transmission Electron Microscopy and Quasi-Elastic Light Scattering Data, Int. J. Biol. Macromol.26 (1999) 145-150.

28. M.A. Sullivan, F. Vilaplana, R.A. Cave, D. Stapleton, A.A. Gray-Weale, R.G. Gilbert, Nature of Alpha and Beta Particles in Glycogen Using Molecular Size Distributions, Biomacromolecules 11 (2010) 1094-100.

29. S.A. Engelberth, N. Hempel, M. Bergkvist, Chemically Modified Dendritic Starch: A Novel Nanomaterial for siRNA Delivery, Bioconjug. Chem. 26 (2015), 1766-1774.

30. F. Lu, Y.Y.C. Mosley, R.J.R. Rosales, B.E. Carmichael, S. Elesela, Y. Yao, H. HogenEsch, Alpha-D-glucan Nanoparticulate Adjuvant Induces a Transient Inflammatory Response at the Injection Site and Targets Antigen to Migratory Dendritic Cells, npj Vaccines 2 (2017) 4.

31. F. Lu, B. Mencia, L. Bi, A. Taylor, Y. Yao, H. HogenEsch, Dendrimer-like Alpha-Dglucan Nanoparticles Activate Dendritic Cells and Are Effective Vaccine Adjuvants, J. Controlled Release 204 (2015) 51-59.

32. S.N. Assen, A. Pospisilova, T.W. Eichler, J. Panek, M. Hruby, P. Stepanek, E. Spiert, D. Jirak, K.O. Skaftnesmo, F. Thorsen, A Novel Nanoprobe for Multimodal Imaging Is Effectively Incorporated into Human Melanoma Metastatic Cell Lines, Int. J. Mol. Sci. 16 (2015) 21658-21680.

33. X. Liang, X. Ren, Z. Liu, Y. Liu, J. Wang, J. Wang, L.M. Zhang, D.Y.B. Deng, D. Quan, L. Yang, An Efficient Nonviral Gene-delivery Vector Based on Hyperbranched Cationic Glycogen Derivatives, Int. J. Nanomedicine 9 (2014) 419-435.

34. Z. Liu, H. Gong, R. Zeng, X. Liang, L.M. Zhang, L. Yang, Y. Lan, Efficient Delivery of NF- $\kappa B$ siRNA to Human Retinal Pigment Epithelial Cells with Hyperbranched Cationic Polysaccharide Derivative-based Nanoparticles, Int. J. Nanomedicine 10 (2015) 2735-2749.

35. S.A. Eccles, G. Bandlow, P. Alexander, Monocytosis Associated with the Growth of Transplated Synergic Rat Sarcomata Differing in Immunogenicity, Br. J. Cancer 34 (1976) 20-27 
36. P. Alexander, S.A. Eccles, C.L.L. Gauci, The Significance of Macrophages in Human and Experimental Tumors, Ann. NY Acad. Sci. 276 (1976) 124-133

37. Q.A. Besford, M. Wojnilowicz, T. Suma, N. Bertleff-Zieschang, F. Caruso, F. Cavalieri, Lactosylated Glycogen Nanoparticles for Targeting Prostate Cancer Cells, ACS Appl. Mater. Interfaces 9 (2017) 16869-16879.

38. P.V. Konarev, V.V. Volkov, A.V. Sokolova, MH.J. Koch, D.I. Svergun, PRIMUS- A Windows-PC Based System for Small-Angle Scattering Data Analysis, J. Appl. Crystallogr. 36 (2003) 1277-1282.

39. T. Masuko, A. Minami, N. Iwasaki, T. Majima, S.I. Nishimura, Y.C. Lee, Carbohydrate Analysis by a Phenol-Sulfuric Acid Method in Microplate Format, Anal. Biochem. 339 (2005) 69-72.

40. S.K. Filippov, O. Sedlacek, A. Bogomolova, M. Vetrik, D. Jirak, J. Kovar, J. Kucka, S. Bals, S. Turner, P. Stepanek, M. Hruby, Glycogen as a Biodegradable Construction Nanomaterial for In Vivo Use, Macromol. Biosci. 12 (2012) 1731-1738.

41. J.D. Fontana, The Presence of Phosphate in Glycogen, FEBS Lett. 109 (1980) 85-92.

42. J. Lomako, W.M. Lomako, W.J. Whelan, R.B. Marchase, Glycogen Contains Phosphodiester Groups That Can Be Introduced by UDPglucose:Glycogen Glucose 1Phosphate, FEBS 329 (1993) 263-267.

43. M. Bertoldo, G. Zampano, L. Suffner, E. Libarti, F. Ciardelli, Oxidation of Glycogen "Molecular Nanoparticles" by Periodate, Polym. Chem. 4 (2013) 653-661.

44. M. Grossutti, J.R. Dutcher, Correlation Between Chain Architecture and Hydration Water Structure in Polysaccharides, Biomacromolecules 17 (2016) 1198-1204.

45. S. Hayashida, Y. Teramoto, T. Inoue, Production and Characteristics of Raw-PotatoStarch-Digesting $\alpha$-Amylase from Bacillus subtilis 65, Appl. Environ Microbiol. 54 (1988) 1516-1522.

46. H.H. Gustafson, D. Holt-Casper, D.W. Grainger, H. Ghandehari, Nanoparticle Uptake: The Phagocyte Problem, Nano Today 10 (2015) 487-510.

47. E. Blanco, H. Shen, M. Ferrari, Principles of Nanoparticle Design for Overcoming Biological Barriers to Drug Delivery, Nat. Biotech. 33 (2015) 941-951.

48. Y. N. Zhang, W. Poon, A.J. Tavares, I.D. McGilvray, W.C.W. Chan, Nanoparticle-Liver Interactions: Cellular Uptake and Hepatobiliary Elimination, J. Controlled Release 240 (2016) 332-348.

49. J.E. Zuckerman, C.H.J. Choi, H. Han, M.E. Davis, Polycation-siRNA Nanoparticles Can Disassemble at the Kidney Glomerular Basement Membrane, PNAS 109 (2012) 3137-3142. 
50. M.A. Jackson, T.A. Werfel, E.J. Curvino, F. Yu, T.E. Kavanaugh, S.M. Sarett, M.D. Dockery, K.V. Kilchrist, A.N. Jackson, T.D. Giorgio, C.L. Duvall, Zwitterionic Nanocarrier Surface Chemistry Improves siRNA Tumor Delivery and Silencing Activity Relative to Polyethylene Glycol, ACS Nano 11 (2017) 5680-5696.

51. Z. Rehman, I.S. Zuhorn, D. Hoekstra, How Cationic Lipids Transfer Nucleic Acids Into Cells and across Cellular Membranes: Recent Advances, J. Controlled Release 166 (2013) 46-56.

52. D. Fischer, Y. Li, B. Ahlemeyer, T. Kissel, In Vitro Cytotoxicity Testing of Polycations: Influence of Polymer Structure on Cell Viability and Hemolysis, Biomaterials 24 (2003) 1121-1131.

53. S.M. Moghimi, P. Symonds, J.C. Murray, A.C. Hunter, G. Debska, A. Szewczyk, A TwoStage Poly(ethylenimine)-Mediated Cytotoxicity: Implications for Gene Transfer/Therapy, Mol. Ther. 11 (2005) 990-995.

54. L.K. Medina-Kauwe, J. Xie, S. Hamm-Alvarez, Intracelullar Reafficking of Nonviral Vectors, Gene Ther. 12 (2005) 1734-1751.

55. L.I. Selby, C.M. Cortez-Jugo, G.K. Such, A.P.R. Johnston, Nanoescapology: Progress Toward Understanding the Endosomal Escape of Polymeric Nanoparticles, WIREs Nanomed. Nanobiotechnol. (2017) e1452.

56. C.E. Zois, A.L. Harris, Glycogen Metabolism Has a Key Role in the Cancer Microenvironment and Provides New Targets for Cancer Therapy, J. Mol. Med. 94 (2016) 137-154.

57. M. Pennati, M. Folini, N. Zaffaroni, Targeting Survivin in Cancer Therapy, Expert Opin. Ther. Targets 12 (2008) 463-476.

58. M. Pennati, M. Binda, M. De Cesare, G. Pratesi, M. Folini, L. Citti, M.G. Daidon, F. Zuino, N. Zaffaroni, Ribozyme-Mediated Down-regulation of Survivin Expression Sensitizes Human Melanoma Cells to Topotecan In Vitro and In Vivo, Carcinogenesis 25 (2004) 11291136.

59. M. Pennati, M. Binda, G. Colella, M. Folini, L. Citti, R. Villa, M.G. Daidone, N. Zaffaroni, Radiosensitization of Human Melanoma Cells by Ribozyme-Mediated Inhibition of Survivin Expression, J. Invest. Dermatol. 120 (2003) 648-654. 


\section{Graphical abstract}

glycogen-siRNA construct

Multicellular Tumor Spheroid

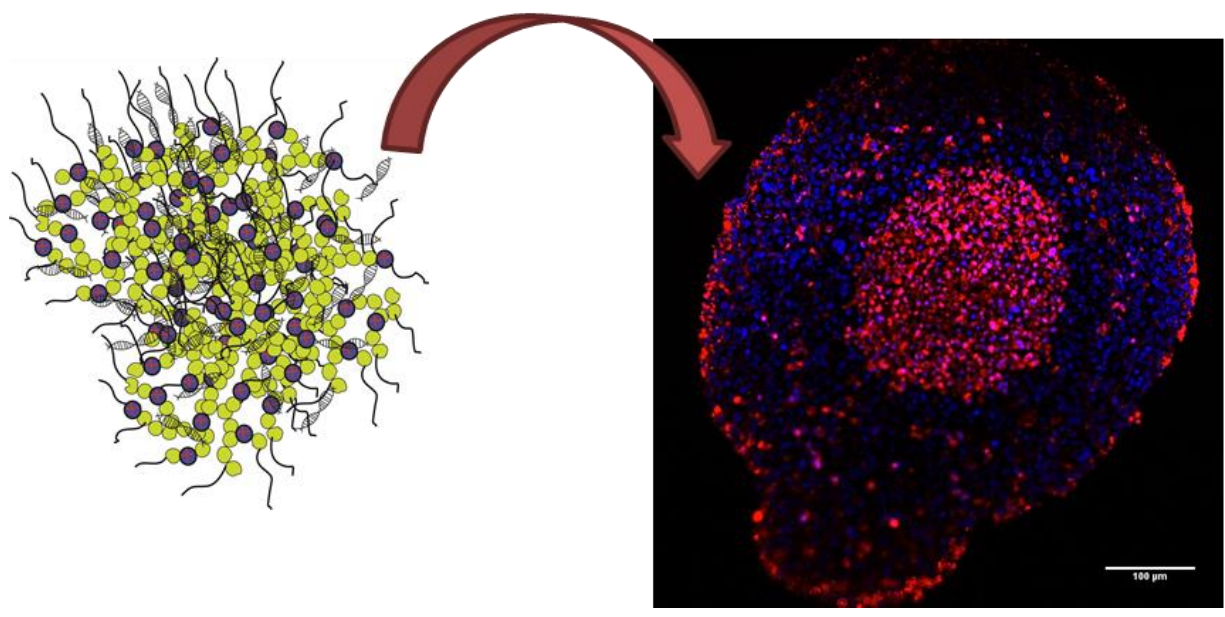

